\title{
Der Markt für Milch und Milcherzeugnisse
}

\author{
Markus Fahlbusch, Anneke Bahr, Bernhard Brümmer und Achim Spiller \\ Georg-August-Universität Göttingen
}

\section{Einleitung}

Das Jahr 2009 zeichnete sich auf dem Milchmarkt in Deutschland und der EU durch insgesamt niedrige Erzeugerpreise aus, die sich allerdings nur zum Teil und verzögert in niedrigeren Verbraucherpreisen widergespiegelt haben. Auch wenn die letzten Monate des Jahres eine Trendwende auf den internationalen Märkten in Richtung steigender Preise gezeigt haben, führte die aus Erzeugersicht unbefriedigende Entwicklung der Milchpreise zu verstärkten Aktionen in Deutschland und der EU, bis hin zu einem erneuten Versuch, einen Milchstreik zu initiieren. Diese Aktionen in der Öffentlichkeit haben dann auch zu diversen Reaktionen auf der politischen Ebene geführt. Von der EU gab es im Oktober nochmals eine Aufstockung der Finanzhilfen in Form eines zusätzlichen Milchfonds, der mittlerweile ein Niveau von 300 Mio. $€$ einnimmt; die Verteilung auf die Mitgliedstaaten folgt dem Anteil an der EU-Milcherzeugung, so dass sich für Deutschland eine Summe von 61 Mio. $€$ ergibt. In Deutschland wurde im Rahmen der Koalitionsverhandlungen ein Sofortprogramm für die Landwirtschaft mit dem besonderen Schwerpunkt Milch festgelegt.

Werden zunächst die mit dem Sofortprogramm des Bundes vereinbarten Elemente betrachtet, so ist ein Gesamtvolumen im Sofortprogramm in Höhe von 750 Mio. $€$ eine beachtenswerte Summe. Diese Gelder sollen in den Jahren 2010 und 2011 zum Einsatz kommen. Es ist vorgesehen, 425 Mio. $€$ in 2010 und die übrigen 325 Mio. $€$ in 2011 zu verteilen. Die Auszahlung dieser Gelder hat dabei gemäß den Vorgaben des Beihilferechts und der EU-Verordnungen zu erfolgen. Entsprechend stellt sich die Umsetzung nicht ganz ohne Schwierigkeiten dar. So sollen im Rahmen der de-minimis-Regelung 85 Mio. $€$ in 2010 in Form einer Kuhprämie ausgezahlt werden. Diese Kuhprämie bezieht sich auf den zu einem Stichtag festgestellten Milchkuhbestand. Nach ersten Berechnungen des BMELV ergibt sich in 2010 ein Betrag von ca. $20 €$ je Kuh, in 2011 wird die Prämie dann aufgrund des geringeren Gesamtvolumens um ca. $12 \%$ geringer ausfallen. Ungeklärt ist zzt. noch, wie mit Betrieben umgegangen werden soll, welche die de-minimis-Ober- grenze (7 $500 €$ in drei Jahren) bereits ausgeschöpft haben.

Als weitere Maßnahme ist eine Grünlandprämie vorgesehen, bei der auf Art. 68 der Betriebsprämienverordnung 73/2009 zurückgegriffen wird. Da hier Obergrenzen über das umzuverteilende und gekoppelt auszuzahlende Finanzvolumen vorgesehen sind, ist die maximal zulässige Summe, die in Deutschland auf diesem Weg umverteilt werden kann, auf 113 Mio. $€$ begrenzt. Das vorhandene Geld soll auf die im Flächenantrag nachgewiesene Grünlandfläche (Dauerund Wechselgrünland) verteilt werden. Damit ergäbe sich ein Betrag von $37 €$ je ha. Aus Sicht der Landwirtschaft erscheint die späte Auszahlung dieser Gelder problematisch. Diese können frühestens mit der Betriebsprämie Ende 2010 ausgezahlt werden. Kurzfristiger einkommenswirksam für die Landwirte sind die Gelder, die für die landwirtschaftliche Unfallversicherung vorgesehen sind. Hier sollen in 2010 und 2011 jeweils 100 Mio. $€$ zur Beitragssenkung zur Verfügung stehen; in 2010 erhöht sich dieser Betrag um nochmals 100 Mio. $€$ aus dem Grünlandmilchprogramm. In 2011 wird diese zusätzliche Summe nur noch 18 Mio. $€$ betragen. In der Summe verbleiben somit noch weitere 25 Mio. $€$ in den beiden Zieljahren. Diese sollen jeweils genutzt werden, um das Liquiditätshilfeprogramm der Landwirtschaftlichen Rentenbank weiter aufrecht zu erhalten.

Es ist aus gesamtwirtschaftlicher Sicht bedauerlich, dass sich der Bund zu einem Maßnahmenbündel entschlossen hat, welches sich samt und sonders (mit Ausnahme der Zinsverbilligung) des Instruments der an den Faktoreinsatz gekoppelten Subventionen bedienen will. Diese Art von Stützungsmaßnahmen ist in verschiedenen wissenschaftlichen Arbeiten bereits als diejenige mit der geringsten Transfereffizienz identifiziert worden. Dadurch, dass ein großer Teil an den kurzfristig vermutlich nur wenig flexiblen Grünlandbestand gekoppelt wird, ist die Transfereffizienz zwar vermutlich etwas besser als beispielsweise in den Studien der OECD unterstellt. Gleichwohl ist die Einkommenswirksamkeit dieser Zahlungen nicht so hoch, wie sie beispielsweise bei einer Aufstockung auf die entkoppelten Betriebsprämien gewesen wäre. Allerdings würde eine solche Aufstockung auch nicht mit 
EU-Recht vereinbar sein. Es zeigt sich deutlich, dass für Feuerwehrmaßnahmen nach dem Motto des Sofortpaketes nur transferineffiziente Wege eingeschlagen werden können. Für die Zukunft lässt sich daraus die Botschaft ableiten, dass ein Mechanismus zur Absicherung gegen „katastrophale“ Marktbedingungen eher auf EU-Ebene verankert werden sollte.

Nach diesem Blick auf die aktuellen politischen Maßnahmen auf dem Milchmarkt werden im folgenden Kapitel die Stufen der Wertschöpfungskette für Milcherzeugnisse zunächst in Deutschland untersucht. Anschließend wird der Blick auf die internationalen Märkte für Milcherzeugnisse gerichtet, hier werden vor allem die internationale Preisbildung, aber auch einige Aspekte auf den EU-Märkten im Mittelpunkt stehen.

\section{Entwicklungslinien in der Wertschöpfungskette}

Im Vordergrund des Jahres 2009 stand die Milchpreisentwicklung. Die EU hat auf die daraus entstandene finanzielle Notlage der Betriebe politisch reagiert, indem sie neben den oben beschriebenen finanziellen Förderungen zwei neue Diskussionspunkte auf die Agenda gesetzt hat. Zum einen wird über die vertraglichen Bindungen zwischen Molkereien und Landwirten und die Förderung von Zusammenschlüssen auf Erzeugerseite beraten. Zum anderen wird die wachsende Konzentration im Lebensmitteleinzelhandel (LEH) kritisch beleuchtet. Als branchenexterne Risiken waren zwei Themen im abgelaufenen Jahr von besonderem Interesse. Für die Reputation der Milchwirtschaft problematisch war das Kennzeichnungswirrwar um Milchimitate und länger haltbare ESL-Milch (Extended Shelf Life). Schließlich brachte das Jahr 2009 insgesamt einen erheblichen Push für den Klimaschutz, ein Thema, das für die Milchwirtschaft aufgrund des Methanausstoßes von Wiederkäuern strategisch bedrohlich ist.

\subsection{Konsumenten}

In dem Jahr 2009 ging der Gesamtkonsum von Milchprodukten deutschlandweit erneut zurück (Tabelle 1). Im Vergleich des zweiten Quartals 2008 mit dem gleichen Zeitraum 2009 errechnet sich ein Preisrückgang für Milchprodukte um $4,8 \%$, gleichwohl ist die konsumierte Menge um 2,3\% gesunken, so dass sich insgesamt ein Umsatzrückgang von $7 \%$ für diesen Zeitraum ergibt. Lediglich bei Butter ist im Jahr 2009 ein leichter Mengenanstieg zu verzeichnen, die Absatzmenge hat aber noch nicht wieder das Niveau aus 2007 erreicht. Der Preisrückgang ist im Übrigen $\mathrm{zu}$ einem erheblichen Teil auf Sonderangebotsaktionen des LEH zurückzuführen (LEBENSMITTELZEITUNG, 2009b: 40).

Ein Mengenrückgang bei gleichzeitig gesunkenen Preisen ist erklärungsbedürftig. Ein Faktor dürfte in der bisher nur begrenzten Weitergabe der Rohstoffpreissenkungen liegen. Da die Erzeugerpreise des Jahres 2009 niedriger waren als in 2007/2008 vor Beginn der hohen Weltmarktpreise, hätten auch die Konsumpreise in diesem Jahr wieder das Niveau vor 2007/2008 erreichen sollen. Nach einem Bericht der EU-KoMMISSION (2009a: 5) lagen die Preise in 2009 aber noch rund $14 \%$ höher als vor 2007/2008. Die EU-KOMMISSION (2009a: 5) schließt daraus, dass die Lebensmittelkette die Rohstoffpreissenkungen nicht vollständig weitergegeben hat (vgl. Kapitel 2.2), was einen Teil der Konsumzurückhaltung erklärt. Auch ist es möglich, dass Verbrauchsgewohnheiten aus der Hochpreisphase beibehalten werden (niedrigpreisige Einkaufsstätten, Handelsmarken, Vermeidung von Verderb usf.) (siehe auch FAHLBUSCH et al., 2008: 37/Abbildung 1). Weiter könnte eine Verunsicherung der Konsumenten durch die öffentliche Diskussion über Milchpreise sowie aufgrund der Kennzeichnungsdiskussion bestehen. Denkbar ist z.B., dass sie Sonderangebote aufgrund von Fairnessüberlegungen meiden, so dass dieses Instrument - wie aus der Praxis berichtet - im Jahr 2009 weniger Wirkungen zeigte. Zum anderen verfügen Milchprodukte grundsätzlich über ein ausgezeichnetes Qualitätsimage beim Verbraucher. Im abgelaufenen Jahr hat aber die irreführende Kennzeichnung von mit Hilfe pflanzlicher Käseersatzstoffe gefertigten Lebensmitteln zu erheblichen Medienreaktionen geführt.

Besonders Käse geriet im Jahr 2009 in den Blickwinkel des Verbraucherschutzes und der Medien.
Tabelle 1. Einkäufe privater Haushalte in Deutschland

\begin{tabular}{|l|r|r|r|c|c|}
\hline Januar bis August & \multicolumn{1}{|c|}{$\mathbf{2 0 0 7}$} & \multicolumn{1}{c|}{$\mathbf{2 0 0 8}$} & \multicolumn{1}{c|}{$\mathbf{2 0 0 9}$} & $\mathbf{\pm \% ~ 2 0 0 9 : 0 8}$ & $\mathbf{\pm \% ~ 2 0 0 9 : 0 7}$ \\
\hline Konsummilch & 2311,4 & 2261,4 & 2226,1 & $-1,6$ & $-3,7$ \\
Butter & 176,0 & 169,7 & 175,3 & $+3,3$ & $-0,4$ \\
Quark & 250,2 & 230,7 & 227,9 & $-1,3$ & $-8,9$ \\
Joghurt & 561,9 & 553,4 & 528,1 & $-4,6$ & $-6,0$ \\
Käse & 539,1 & 520,2 & 518,1 & $-0,4$ & $-3,9$ \\
\hline
\end{tabular}

Angaben in Mio. 1/1 $000 \mathrm{t}$

Quelle: DeUTSChE MiLchwirTSChaft (2009: 817) 
Abbildung 1. Milcherzeugerpreis und Endverbraucherpreise verschiedener Milchprodukte

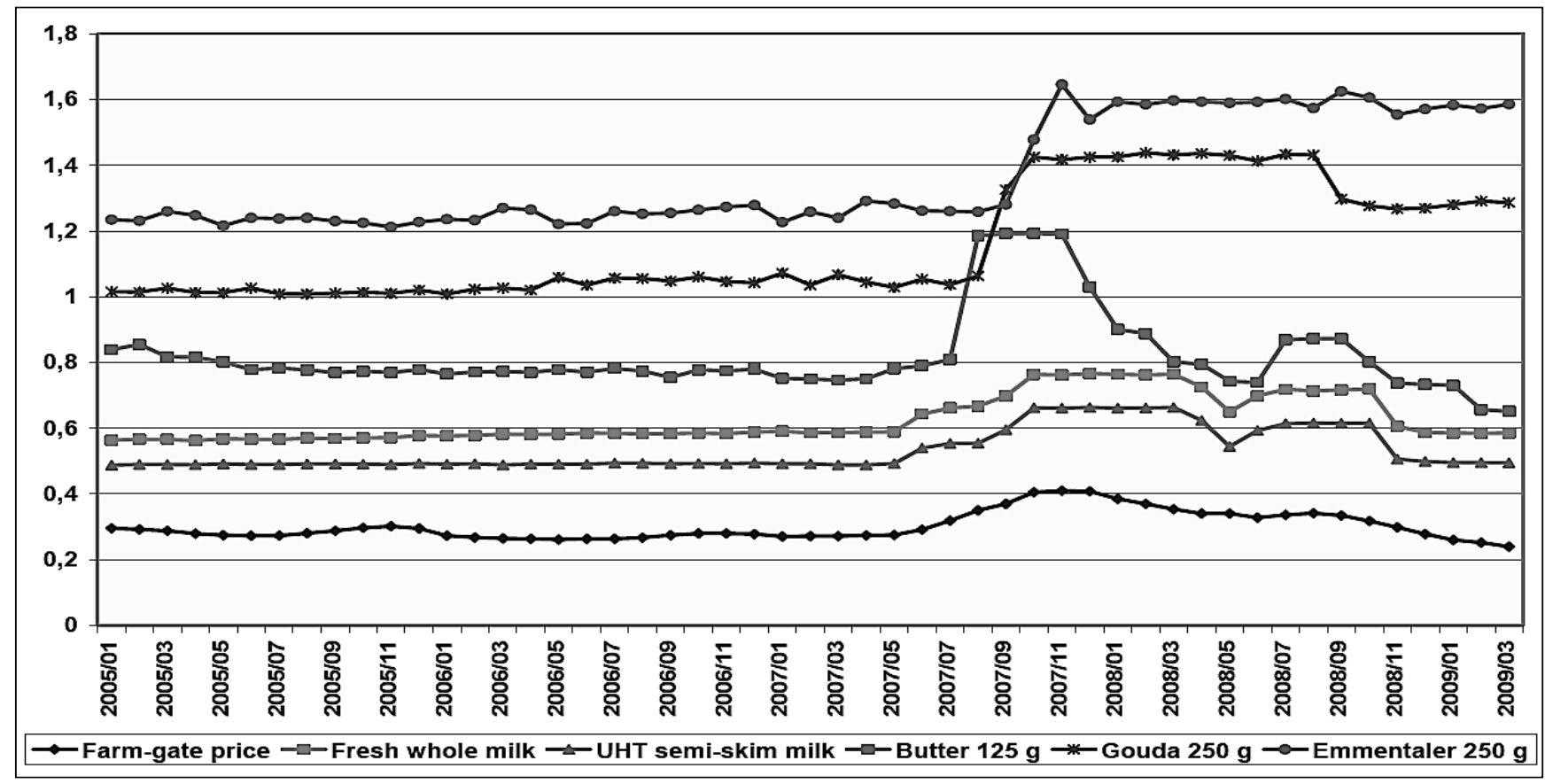

Quelle: EU-KoMmission (2009d: 35)

Bereits vor gut 20 Jahren stellten MEYER und WEINDLMAIER (1988: 66) in einer Studie zu Milchimitaten fest, dass diese von einem Teil der Verbraucher bewusst aus gesundheitlichen Gründen konsumiert werden, insbesondere aber für Großverbraucher eine preisgünstige Alternative darstellen können. Die Autoren weisen bereits auf die Relevanz einer eindeutigen Kennzeichnung hin, da die Verbraucher sich bei unzureichender Auskunft getäuscht fühlen könnten. Gleichzeitig schätzten sie das Substitutionspotenzial in der Lebensmittelindustrie als beachtlich hoch ein.

Die in Tabelle 2 dargestellten Ergebnisse von Kontrollen der zuständigen Überwachungsbehörden im Jahr 2008 lassen erkennen, dass irreführende Kennzeichnung nicht erst im abgelaufenen Jahr ein virulentes Problem darstellte. Gut $30 \%$ bis fast $60 \%$ der Beanstandungen entfallen auf falsche Deklarierung. Hierzu zählen allerdings auch Haltbarkeitsdaten

Tabelle 2. Anzahl der Kontrollen und Beanstandungen bei Käse in 2008

\begin{tabular}{|l|r|r|r|}
\hline Bundesland & $\begin{array}{c}\text { Proben- } \\
\text { zahl }\end{array}$ & $\begin{array}{c}\text { Beanstandete } \\
\text { Proben }\end{array}$ & Irreführend $^{\mathbf{1})}$ \\
\hline Niedersachsen & 987 & $203(20,6 \%)$ & $72(35,5 \%)$ \\
Nordrhein-Westfalen & 306 & $28(9,2 \%)$ & $16(57,1 \%)$ \\
Bayern & 2229 & $226(10,1 \%)$ & $99(43,8 \%)$ \\
\hline
\end{tabular}

1) nach § 11 LFGB und Art. 16 EG 178/2002

Quelle: eigene Darstellung nach den Jahresberichten 2008 der drei Landesämter für Lebensmittelüberwachung und andere Aufdrucke auf der Verpackung. Als Käse dürfen nur solche Produkte benannt werden, die aus Milch hergestellt sind (MILCHINDUSTRIEVERBAND, 2009: 46). Ganz offensichtlich liegen aber Probleme der Rechtsdurchsetzung vor (LEBENSMITTELZEITUNG, 2009a: 24). Im Gegensatz dazu ging es bei der Kennzeichnung von ESL- als Frischmilch um Fragen der Klarheit von Produktbezeichnungen im Falle innovativer Technologien, die zu einer neuen Produktkategorie führen. Die Milchwirtschaft musste hier zur Kenntnis nehmen, dass zwar die überwiegende Zahl der Verbraucher die Änderung nicht wahrgenommen hat, aber auch kleinere Gruppen Ansprüche an eine transparente Produktkennzeichnung legitimieren können. Gleichzeitig zeigt aber der Markterfolg der ESLMilch, dass im Hinblick auf die beiden zentralen, sich aber z.T. widersprechenden Kaufmotive Frische und Convenience in einer zeitknappen Gesellschaft letzteres langfristig an Bedeutung gewinnt.

\subsection{Lebensmitteleinzelhandel}

Der niedrige Milchauszahlungspreis des Jahres 2009 hat auf verschiedenen Ebenen Diskussionen um potenzielle Ursachen ausgelöst. Neben dem Marktungleichgewicht wird dabei in Politik wie Wirtschaft immer wieder der Blick auf die Struktur und das Marktverhalten des Lebensmitteleinzelhandels geworfen. Angesichts der im internationalen Vergleich herausgehobenen 
Wettbewerbsposition der deutschen Discounter und einer Konzentration von rund $90 \%$ des LEH-Umsatzes auf die sechs größten Unternehmen gewinnt die Auseinandersetzung um die Nachfragemacht an Brisanz. Kern der Frage ist eine mögliche asymmetrische Preistransmission zu Lasten der vorgelagerten Wertschöpfungsstufen (BARROS et al., 2006). Abbildung 1 zeigt am Beispiel von fünf Milchprodukten, dass der Erzeugerpreis nur bedingt mit dem Endverbraucherpreis korreliert und speziell der Käsepreis zeitverzögert reagiert (EU-KOMMISSION, 2009d: 35f.). Die Preise für Molkereiprodukte werden zwischen Industrie und Handel teilweise jährlich (Markenware, höher veredelte Spezialitäten), seit 2007/08 aber auch halbjährlich (Handelsmarken, Commodities) bzw. monatlich (Butter) verhandelt. Die stärkere Rigidität des Käsepreises ist damit gut erklärbar. Mangels belastbarer Datenlage offen bleiben muss aber die letztlich entscheidende Frage, ob Preisanhebungen des Handels schneller als Preissenkungen erfolgen und ob über einen längeren Zeitraum die Handelsspanne zu Lasten der Produzenten größer geworden ist.

Als Reaktion auf den Vorwurf der Nachfragemacht hat der Hauptverband des Deutschen Einzelhandels (HDE) eine Studie zur Struktur der IndustrieHandels-Beziehungen in Auftrag gegeben (IFH und BBE, 2009). Kern des Gutachtens ist die warengruppenvergleichende Analyse von drei zentralen Merkmalen der Geschäftsbeziehung: 1. Anteil des Lebensmitteleinzelhandels am Absatz der Lebensmittellieferanten im Vergleich zum Vertrieb über AußerHaus-Markt, Industriekundengeschäft, Handwerk und Exportgeschäft; 2. Vergleich der Konzentration auf Industrie- bzw. Handelsseite; 3. Vergleich von Markentreue versus Geschäftsstättentreue der Verbraucher. Die vom Institut für Handelsforschung an der Universität Köln und der Unternehmensberatung BBE Retail Experts erarbeitete Studie zeigt für die Molkereiindustrie im Vergleich zu fast allen anderen Warengruppen der Ernährungsindustrie eine ausgesprochen schlechte Verhandlungsposition.

Hinsichtlich der Vertriebsalternativen der deutschen Molkereien wird deutlich, dass 35,1\% der Milchproduktion exportiert werden, wobei ein beachtlicher Anteil dieses Exportes über deutsche Discounter im Ausland erfolgt, da diese heimische Lieferanten auch für einen Teil ihrer Auslandsmärkte nutzen (FAHLBUSCH et al., 2009: 39). Insofern verringert die Exportaktivität der Milchindustrie die Abhängigkeit von den Großabnehmern des LEH nur bedingt. Bezogen auf den inländischen Markt entfallen $82 \%$ des Molkerei-Umsatzvolumens (Nettobeschaffungswert) auf den institutionellen Lebensmitteleinzelhandel, $6 \%$ auf andere Vertriebswege (Fachhandel, Kiosk usf.) und $12 \%$ auf den Außer-Haus-Markt (Gastronomie, Großverbraucher). Damit gehört die Molkereiwirtschaft zu den Branchen mit der stärksten Konzentration des Absatzes auf den LEH (Spannweite von $36 \%$ bei Tabakwaren bis $91 \%$ bei Hygiene/Papier). Hinzu kommt, dass der Discountanteil bei Milchprodukten im Vergleich $\mathrm{zu}$ vielen anderen Warengruppen mit durchschnittlich $54 \%$ der Menge sehr hoch liegt (vgl. Tabelle 3, Umsatzanteil ca. 42\%, IFH und BBE, 2009).

\section{Tabelle 3. Absatzanteile der Discounter bei Milchprodukten 2008}

\begin{tabular}{|l|c|}
\hline Produkt & Marktanteil \\
\hline Konsummilch & $56 \%$ \\
Käse & $55 \%$ \\
Quark & $55 \%$ \\
Joghurt & $55 \%$ \\
Butter & $52 \%$ \\
Milchgetränke & $49 \%$ \\
Durchschnitt der Produkte & $54 \%$ \\
\hline
\end{tabular}

Quelle: ZMP, zitiert nach MEINE MiLCH (2009)

Betrachtet man das Beschaffungs- bzw. Absatzvolumen der jeweils führenden fünf Händler/Hersteller, so nimmt die Molkereiindustrie eine vergleichsweise schlechte Position gegenüber dem Handel ein, da hier die Herstellerkonzentration mit rund $40 \%$ deutlich geringer ist als die Beschaffungskonzentration auf Handelsseite mit über $60 \%$ (vgl. Abbildung 2). Hersteller- wie Handelskonzentration sind im Übrigen bei der Weißen Linie etwas höher als in der Gelben Linie. Zudem weist die Sektoruntersuchung Milch des BUNDESKARTELLAMTES (2009b: 36) auf die starke Konzentration des LEH hin. Rund $90 \%$ der Markanteile entfallen auf die größten sechs Handelsunternehmen (Edeka, Rewe, Aldi, Lidl (inkl. Kaufland), Metro und Tengelmann) (BUNDESKARTELLAMT, 2009b: 36). Der LEH hat außerdem durch die hohe Preistransparenz in der Kette (transparente Milcherzeugerpreise) Vorteile gegenüber den vorgelagerten Stufen (BUNDESKARTELLAMT, 2009b). Außerdem hat das BUNDESKARTELLAMT (2009b) eine Befragung von Molkereien durchgeführt. Danach sehen sich $60 \%$ der Molkereien durch die Nachfragemacht des LEH geschwächt, wobei sich die Position von Commodity-Herstellern schwieriger darstellt als für Markenartikelproduzenten (BUNDESKARTELLAMT, 2009b: 99). Zudem existiert eine hohe Abhängigkeit bei einigen Molkereien von nur einer Han- 

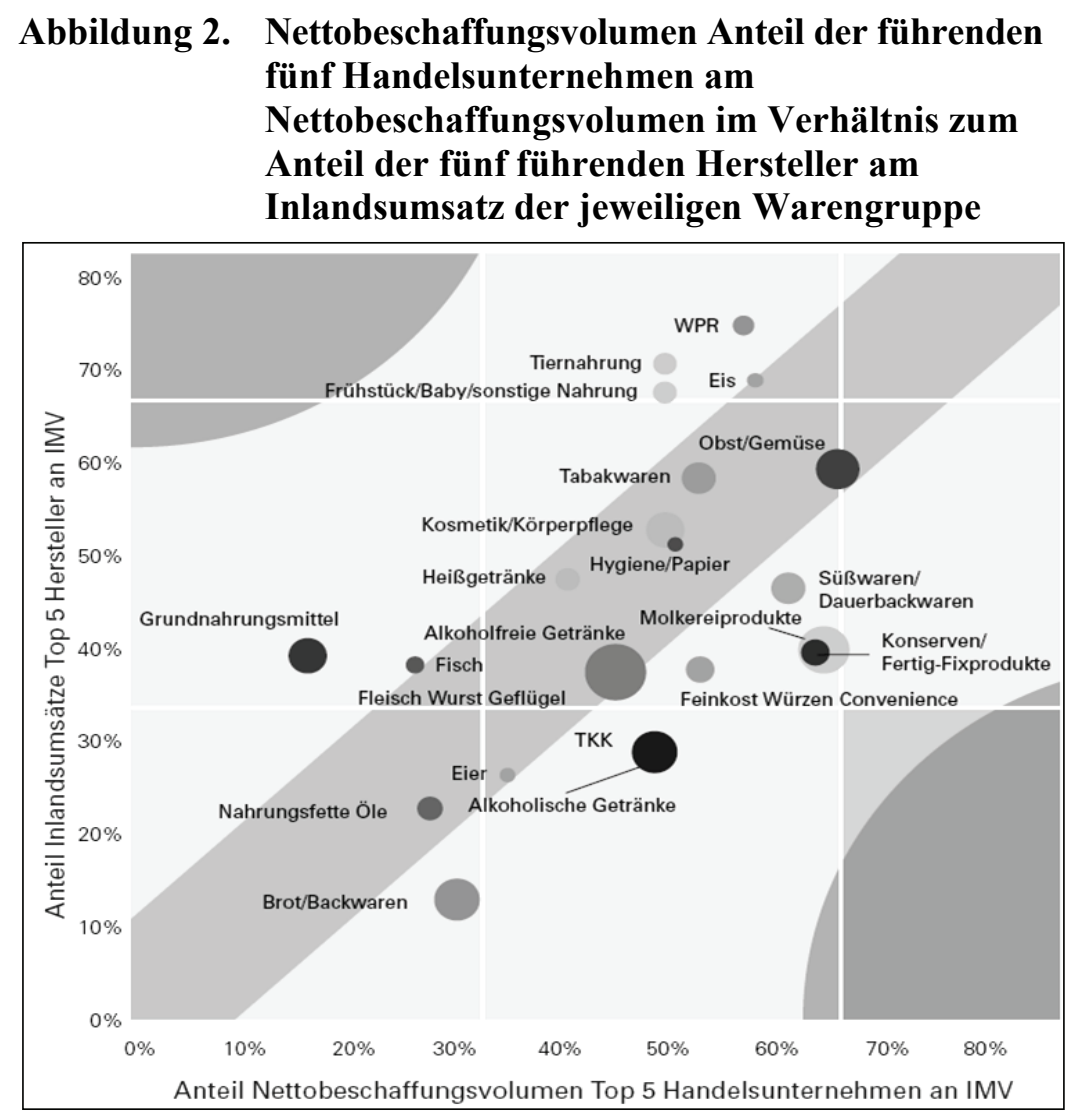

Quelle: IFH UND BBE (2009)

delskette. Für Molkereien, die zwischen 30-50 \% des Absatzes über einen Abnehmer (insb. Lidl) realisieren, wäre ein Verlust dieses Handelspartners wirtschaftlich schwierig zu verkraften (BUNDESKARTELLAMT, 2009b: 98).

Im dritten Analyseschritt der IFH/BBE-Studie wurde schließlich im Rahmen einer Konsumentenbefragung erhoben, bei welchen Lebensmitteln die Verbraucher ausgeprägte Markenpräferenzen aufweisen und bei Auslistung einer Marke die Einkaufsstätte wechseln würden. Auch hier zeigt sich, dass die beiden untersuchten Milchprodukte (Joghurt und Frischkäse, d.h. Milchprodukte mit eher höherer Markenrelevanz) im Vergleich zu anderen Lebensmitteln durch eine geringere Markentreue charakterisiert sind. Nur rund $25 \%$ der Befragten präferieren bei diesen Milcherzeugnissen eine bestimmte Marke, die übrigen Befragten wechseln eher die Marke als das Geschäft. Diese geringe Markentreue schlägt sich auch in dem hohen Marktanteil der Handelsmarken nieder, der beispielsweise $82,3 \%$ des Umsatzes bei H-Milch, $70,8 \%$ bei Frischmilch und 56,2 \% bei Quark beträgt. Insgesamt liegt der Umsatzanteil der Handelsmarken in 2008 bei 56,6 \% (Weiße Linie) bzw. 48,9 \% (Gelbe Linie) (MEIER, 2009). Bezogen auf die führenden Handelsunternehmen beträgt am Beispiel der H-Milch der Handelsmarkenanteil $100 \%$ bei Aldi, $93 \%$ bei Lidl, $85 \%$ bei Rewe und $68 \%$ bei Edeka (BOSCHET, 2009). Ein Handelsmarkenspezialist wie die Milch-Union Hocheifel (MUH) produziert $\mathrm{zu}$ rund $85 \%$ Handelsmarken. Trotzdem liegt der Marktanteil der MUH bei H-Milch unter $15 \%$ (ähnlich Müller-Milch, Marktführer ist NordContor mit knapp 25\% Marktanteil (LEBENSMITTELZEITUNG, 2009d).

Insgesamt ergeben sich fundierte Hinweise auf die vergleichsweise gute Verhandlungsposition des Lebensmittelhandels bei Milchprodukten, die sich in einem hohen Preisdruck und in einer weitgehenden Austauschbarkeit der Lieferanten niederschlägt.

\subsection{Molkereiwirtschaft}

Die Molkereien sahen sich im Jahr 2009 deutlich schwierigeren Marktumständen gegenüber. Durch die hohen Milchpreise in den Jahren 2007 und 2008 sind viele Absatzmärkte eingebrochen und haben sich trotz der Preissenkungen nur schleppend erholt. Der Milchindustrieverband vermeldet für den Zeitraum von Januar 2009 bis Juli 2009 einen Rückgang des Branchenumsatzes um $17 \%$ (LEBENSMITTELZEITUNG, 2009c).

Nach Marktberichten konnte besonders das Industriekundengeschäft nicht mehr im Umfang der Vorjahre realisiert werden. Viele weiterverarbeitende Unternehmen haben aufgrund der hohen Milchpreise der Vorjahre ihren Rohstoffbedarf durch Substitute aus pflanzlichen Rohstoffen gedeckt. Die Erfahrungen des aktuellen Jahres zeigen, dass diese aufgrund der hohen Umstellungskosten die Produktion nur bei deutlich veränderten Preisrelationen wieder auf Milchrohstoffe umstellen und entsprechend preisunelastisch reagieren (DEUTSCHE MOLKEREI ZEITUNG, 2009). Entsprechend werben beispielsweise Unternehmen wie Cargill, die Raffinerien für pflanzliche Fette betreiben, für Käseimitate vollständig aus pflanzlichen Rohstoffen, um damit unabhängig von den Preisvolatilitäten des Milchmarktes zu sein (IDM, 2009b). Andererseits ist der Marktanteil von Käseimitaten relativ niedrig und beträgt nur ca. $1 \%$ des gesamten Käses in Deutschland (KECKL, 2009).

Der Mengen- und Preisdruck und die oben geschilderte schwierige Verhandlungsposition der In- 
Tabelle 4. Ranking der führenden deutschen Molkereien nach Umsatz in 2008 und 2007

\begin{tabular}{|l|l|c|c|}
\hline Unternehmen & $\begin{array}{c}\text { Umsatz 2008 } \\
\text { (Mio. } € \text { ) }\end{array}$ & $\begin{array}{c}\text { Umsatz 2007 } \\
\text { (Mio. } \mathbf{~} \text { ) }\end{array}$ \\
\hline 1 & $\begin{array}{l}\text { Nord-Contor } \\
\text { Milch }^{1)}\end{array}$ & 4600 & $\begin{array}{c}\text { NM: } 2300 ; \\
\text { Humana: } 2200\end{array}$ \\
2 & Müller $^{\text {S }}$ & 2300 & 2200 \\
4 & Hochwald & 1250 & 1100 \\
5 & Hochland & 1160 & 1000 \\
6 & FrieslandCampina & 910 & Campina: 869 \\
7 & Zhrmann & 650 & 650 \\
8 & Mutt & 620 & 700 \\
9 & Bayernland & 619 & 547 \\
10 & Danone & 610 & - \\
\hline
\end{tabular}

1) NordContor ist die gemeinsame Vertriebsorganisation von Humana und Nordmilch, $\mathrm{S}=$ Schätzung

Quelle: MOLKEREIINDUSTRIE (2009), Auskünfte der Unternehmenshomepage

dustrie gegenüber dem LEH führte zu weiteren Unternehmenszusammenschlüssen in 2009 , so dass sich das Ranking der führenden Molkereien deutlich verändert hat. Die größte Beachtung hat die inzwischen vom Bundeskartellamt genehmigte strategische Vertriebsallianz der Unternehmen Nordmilch AG und der Humana $\mathrm{GmbH}$ gefunden, die jetzt auf Basis einer gemeinsamen Vertriebsorganisation und der jeweiligen Quotenmengen ihr Geschäft steuern. Da eine Fusion der Unternehmen für 2010 wahrscheinlich ist, betrachten wir im folgenden Ranking beide Unternehmen gemeinsam (Tabelle 4). Des Weiteren konnte sich die Nordmilch durch den Zusammenschluss mit der Dargun eG Pommernmilch (311 Mio. kg von 231 Mitgliedern (CORDES, 2009)) eine Lieferantenbasis in Mecklenburg-Vorpommern sichern. Außerdem stimmte das Bundeskartellamt dem Zusammenschluss der Humana Milchunion eG und der Milchveredelung Niedergrafschaft eG zu. Der Käsespezialist Hochland übernahm 2009 den Käsehersteller Grünland (BUNDESKARTELlamT, 2009a). Auch der Strukturwandel auf der Ebene der Produktionsstätten setzt sich weiter fort. Durch einen Neustrukturierungsprozess will die niederländische Genossenschaftsmolkerei Royal FrieslandCampina 6 Werke in Deutschland, Holland und Belgien schließen und ihre Produktionsprozesse dadurch neu ausrichten (AGRARZEITUNG, 2009). Insgesamt steigt FrieslandCampina auf Platz fünf der deutschen Umsatzliste auf. Die Molkerei Meggle scheidet aus den Top 10 aus, dafür ist Danone in das deutschlandweite Ranking eingestiegen.
Bei einem internationalen Vergleich der Konzentrationsraten $\left(\mathrm{CR}_{3}\right)$ wird deutlich, dass die größten drei Molkereiunternehmen (Nordmilch AG, Müller und Humana $\mathrm{GmbH}$ ) mit einer Verarbeitung von $35 \%$ des nationalen Milchanfalls im europäischen Vergleich geringere Marktanteile auf sich vereinigen $\left(\mathrm{CR}_{3}\right.$ : UK $41 \%$, Frankreich $49 \%$; Niederlande $90 \%$, Skandinavien fast 100\%) (INDERHEES, 2009: 548). Durch die Gründung des NordContor ergibt sich ein neues Bild, da der Verbund allein $23 \%$ der deutschen Milchmenge bündelt (JANSHEN, 2009) und damit als erstes deutsches Unternehmen in die Top 10 des weltweiten Rankings aufsteigt (Tabelle 5). Unternehmensgröße ist zudem nur ein Indikator für Wettbewerbsfähigkeit. Hinsichtlich der Größe der Betriebsstätten und damit der produktionstechnischen Skalenvorteile steht die deutsche Molkereiindustrie gar nicht schlecht da: Der Anteil der Fabriken mit mehr als $100000 \mathrm{t}$ Jahresproduktion lag auf Basis einer EUROSTAT-Auswertung für das Jahr 2006 bei 32,6\% und damit ähnlich wie in Belgien (32,8 \%), während in der EU-27 nur 3,9\% diese Größenschwelle überschreiten (Polen 8,4\%, Frankreich 5,7\%, UK 4,4\%, Italien $0,7 \%$; EUROSTAT, 2008). Branchenschätzungen gehen davon aus, dass für eine wettbewerbsfähige Commodity-Produktion mindestens Betriebsgrößen von ca. $1 \mathrm{Mrd}$. $\mathrm{kg}$ pro Jahr notwendig sind.

Vor dem Hintergrund der auslaufenden politischen Mengensteuerung hat im Jahr 2009 eine verstärkte Diskussion um eine Neugestaltung der Lieferbeziehungen zwischen Molkereien und ihren Lieferantenbegonnen (WEINDLMAIER und OBERSOJER 2009; STEFFEN et al, 2009). Auch die EU hat die Vertragsstrukturen zwischen Molkereien und Landwirten in ihrem Sechs-Punkte-Plan in den Vordergrund gestellt. Im Gegensatz dazu besteht allerdings in der wissenschaftlichen Diskussion Einigkeit darüber, dass zwar bei

\section{Tabelle 5. Führende Molkereiunternehmen weltweit nach Umsatz in 2008/2009}

\begin{tabular}{|l|l|c|}
\hline & Unternehmen & $\begin{array}{c}\text { Umsatz ausMilch } \\
\text { (Mrd. } € \text { ) }\end{array}$ \\
\hline 1 & Nestlé (CH) & 18,5 \\
2 & Danone (Fr) & 10,7 \\
3 & Lactalis (Fr) & 9,3 \\
4 & FrieslandCampina (NL) & 9,3 \\
5 & Fonterra (NZ) & 8,2 \\
6 & Dean Foods (US) & 8,1 \\
7 & DFA (US) & 6,9 \\
8 & Arla Foods (Den/Swe) & 6,9 \\
9 & Kraft Foods (US) & 5,1 \\
10 & Nord-Contor Milch (Ger) & 4,7 \\
\hline
\end{tabular}

Quelle: IDM (2009a) 
einem Wegfall der einzelbetrieblichen Mengengrenzen neue Vertrags- und Preiskonstellationen gerade in den Genossenschaften gefunden werden müssen (Tabelle 6), privatwirtschaftliche Vertragsklauseln jedoch kein Ersatz für die Milchquote, sondern ein Instrument des einzelbetrieblichen Beschaffungsmanagements sind. Verschiedene Molkereien in Österreich und Deutschland haben in diesem Rahmen Sanktionssysteme für Überlieferungen der Landwirte eingeführt. So hat beispielsweise die Berglandmilch in Österreich ein monatliches Preiszu- bzw. -abschlagssystem entwickelt, bei dem in Monaten mit hohem Milchaufkommen ein Abschlag auf die überlieferte Milch vorgenommen wird. In Monaten mit geringem Milchaufkommen werden Zuschläge gezahlt, wenn die betriebliche Jahresquote noch nicht ausgeschöpft ist (ALTMANN, 2009). Auch die deutsche Upländer Bauernmolkerei zahlt für überlieferte Bio-Milch lediglich den Spot-Markt-Preis. Ein Teil der Unternehmen fährt damit eine Beschaffungsstrategie, bei der nur für Milch, die innerhalb der Quote geliefert wird, der volle Preis gezahlt wird, bei Überlieferung dagegen der am Markt erlösbare Preis zählt (ALTMANN, 2009). Dagegen kommunizieren Privatmolkereien wie Zott, Jäger oder Bergader und Genossenschaften wie die Nordmilch eine unbegrenzte Abnahmegarantie für die Milchmenge ihrer festen Vertragslieferanten (z.T. wurden Verträge mit mehr als fünf Jahren Laufzeit geschlossen) bzw. Genossenschaftsmitglieder und setzen damit Wachstums- und Bindungsanreize. Der gleichzeitige Versuch der Nordmilch, durch ein gerichtliches Vorgehen gegen Mitgliederkündigungen via Rechtsformenwechsel die Langfristigkeit der Geschäftsbeziehungen zu sichern, war allerdings juristisch nicht erfolgreich (LEBENSMITTELZEITUNG, 2009e), zeigt aber, dass viele Genossenschaften zzt. eine Konzentration ihrer Beschaffung auf langfristige Mitgliedslieferanten vornehmen.

Tabelle 6. Anteil der Genossenschaften an der Milchverarbeitung in verschiedenen Ländern Europas

\begin{tabular}{|l|c|}
\hline Land & Anteil Genossenschaften \\
\hline Finnland & $96 \%$ \\
Dänemark & $95 \%$ \\
Österreich & $94 \%$ \\
Niederlande & $83 \%$ \\
Portugal & $82 \%$ \\
Irland & $62 \%$ \\
Deutschland & $60 \%$ \\
Italien & $38 \%$ \\
\hline
\end{tabular}

Quelle: JANSHEN (2009)

\subsection{Landwirtschaft}

Für die Landwirte war das Jahr 2009 durch den sinkenden Milchpreis geprägt, der durch verschiedene Einflüsse (s.u.) im Sommer 2009 teilweise unter das Interventionspreisniveau von $21 \mathrm{Cent} / \mathrm{kg}$ gesunken ist (vgl. Abbildung 3). Der durchschnittliche europäische Milchpreis für das erste Halbjahr 2009 lag bei 24 Cent/kg (2007/2008: 30-40 Cent/kg) (EU-KoMMISSION, 2009a: 2). Durch diese Entwicklung ergaben sich teilweise Liquiditätsengpässe auf den Betrieben. Zusätzlich wurden in manchen Milchviehbetrieben Steuernachzahlungen aus dem hochpreisigen Jahr 2007 notwendig. Dies brachte einige Betriebe in eine noch größere Schieflage.

\section{Abbildung 3. Milcherzeugerpreise in Deutschland 2007-2009}

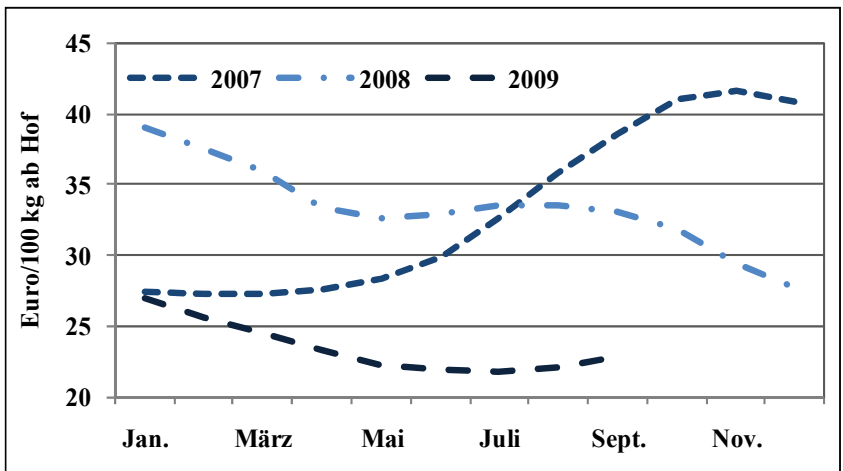

Quelle: eigene Darstellung nach ZMP (2008), BMELV (2009) und AMI (2009)

Die Bundesländer haben in Zusammenarbeit mit der Rentenbank ein Soforthilfe-Kreditprogramm gestartet, das eine einprozentige, durch den Bund finanzierte Zinsverbilligung vorsieht. Das Darlehen wird für vier Jahre gewährt und kann im ersten Jahr tilgungsfrei sein (RENTENBANK, 2009a). Der Zuspruch der Landwirte war groß. Schon nach kurzer Zeit konnte die Rentenbank melden, dass ein Drittel der Kreditsumme bereits vergeben war, zwei Drittel davon an Milchviehbetriebe (RENTENBANK, 2009b).

Diese Art der Finanzierung löst aber keine Rentabilitätsprobleme der Unternehmen und verleitet möglicherweise einige Betriebsleiter, die eigentlich notwendige Aufgabe unrentabler Betriebe hinauszuzögern.

Der Strukturwandel in der Landwirtschaft spiegelt die Preisentwicklung der letzten Jahre (Tabelle 7) wider. Wurden im Jahr 2008 erstmals (fast) keine Betriebe mehr aufgegeben, zeigte sich in 2009 wieder die aus den Vorjahren bekannte Abschmelzungsrate, 
wobei verschiedene Studien auf die beachtlichen regionalen Unterschiede hinweisen (LASSEN et al., 2009; Rothfuß et al., 2009). Die Anzahl der Milchviehhalter ist in Deutschland in 2009 auf unter 100000 gesunken. Ein eindeutiges Indiz für die kurzfristigen Reaktionen der Landwirte auf das Preisniveau ist auch die Anzahl der gehaltenen Milchkühe, die in 2008 ein Rekordhoch erreichte. In 2009 zeichnet sich eine Reduzierung des Milchkuhbestandes ab. Die Milchleistung je Kuh war in 2008 leicht rückläufig. Verbunden mit dem hohen Milchpreis lässt sich vermuten, dass auch Kühe mit einer schlechteren Leistung noch in der Produktion gehalten wurden. Dies ist in 2009 nicht der Fall. Ein Indiz hierfür sind die derzeit hohen Aufkommen an Schlachtkühen, was sich negativ auf deren Preise auswirkt (07/2008: 2,77€/kg; 10/2009: 2,10€/kg) (AMI, 2009).

Ein weiterer Grund für die Beendigung der Produktion zum gegenwärtigen Zeitpunkt kann, wie auch schon in dem vergangenen Jahr, darin liegen, dass die Betriebsleiter die Möglichkeit nutzen, ihr in der Milchquote gebundenes Kapital vor dem Auslaufen der Quote zu liquidieren. Die betriebliche Quotenmenge kann ebenfalls eine Begründung dafür sein, dass verstärkt Kühe aus der Produktion genommen werden, so dass Betriebe, die nicht ausreichend Menge absichern können, eher Kühe schlachten lassen, als sich neue Kapazitäten durch Quotenzukauf zu schaffen.

Eine interessante Entwicklung ergab sich in diesem Jahr an der Milchquotenbörse. Konnte bei dem Termin im Juli 2009 ein Tiefpreis mit 14 Cent $/ \mathrm{kg}$

Tabelle 7. Strukturwandel der deutschen landwirtschaftlichen Betriebe

\begin{tabular}{|c|c|c|c|c|c|}
\hline & $\begin{array}{l}\text { Milchkuh- } \\
\text { bestand } \\
\text { in } 1000\end{array}$ & $\begin{array}{c}\text { Anzahl } \\
\text { Milchvieh- } \\
\text { halter in } \\
1000\end{array}$ & $\begin{array}{c}\text { Anzahl } \\
\text { Kühe } \\
\text { pro } \\
\text { Betrieb }\end{array}$ & $\begin{array}{c}\text { Milch- } \\
\text { produktion } \\
\text { pro Betrieb } \\
\text { in } \mathrm{t}\end{array}$ & $\begin{array}{l}\text { Milch- } \\
\text { ertrag } \\
\text { je Kuh }\end{array}$ \\
\hline 1990 & 6355 & 278 & 22,9 & 114 & 4857 \\
\hline 1995 & 5229 & 196 & 26,7 & 146 & 5427 \\
\hline 2000 & 4564 & 136 & 33,6 & 205 & 6122 \\
\hline 2005 & 4164 & 110 & 37,9 & 256 & 6761 \\
\hline 2006 & 4054 & 106 & 38,2 & 262 & 6849 \\
\hline 2007 & 4087 & 101 & 40,4 & 281 & 6944 \\
\hline 2008 & 4230 & 101 & 41,9 & 287 & 6827 \\
\hline $2009^{*}$ & 4210 & 97 & 43,4 & $* *$ & $* *$ \\
\hline \multicolumn{6}{|c|}{ Ø Veränderung pro Jahr in \% } \\
\hline $\begin{array}{l}1990- \\
2009\end{array}$ & $-2,1$ & $-5,4$ & 3,5 & $\begin{array}{c}5,3 \\
\text { (ohne 2009) }\end{array}$ & $\begin{array}{c}1,9 \\
\text { (ohne 2009) }\end{array}$ \\
\hline
\end{tabular}

* vorläufig;** nicht verfügbar

Quelle: eigene Zusammenstellung nach ZMP (verschiedene Jahrgänge) und AMI (2009), eigene Berechnungen
Abbildung 4. Quotenmenge und Preise in Deutschland

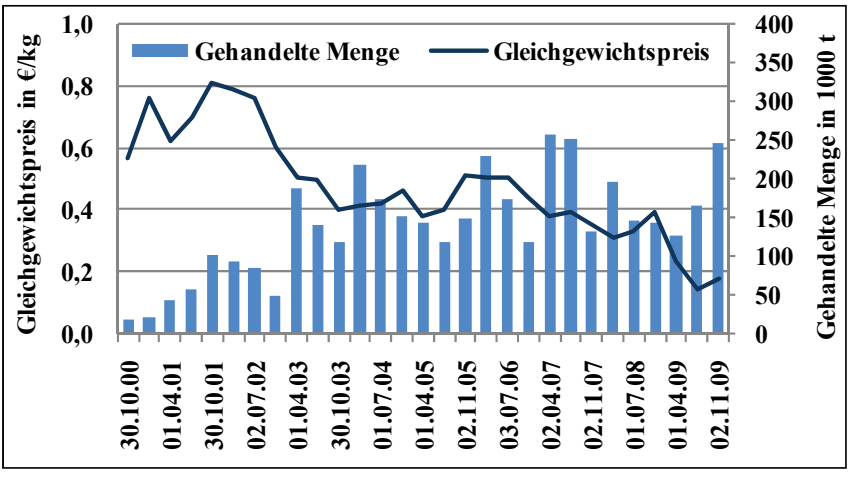

Quelle: eigene Darstellung nach ZMP (2008), DBV (2009) und AMI (2009)

(Gleichgewichtspreis) ausgemacht werden, ist im November der Gleichgewichtspreis für das gesamte Bundesgebiet auf 18 Cent/kg angestiegen (vgl. Abbildung 4). Besonders die westdeutsche Börse hatte mit $20 \mathrm{Cent} / \mathrm{kg}$ hierauf Einfluss (Ostdeutschland: $11 \mathrm{Cent} / \mathrm{kg}$ ). Zwar sind tendenziell die Quotenpreise im Herbst höher, da die Betriebe dann abschätzen können, inwiefern die betriebliche Quotenmenge ausreicht. Außerdem können Meldungen über eine fast hundertprozentige Ausnutzung der Quote in den ersten Monaten des Milchwirtschaftsjahres zu einer Verunsicherung geführt haben. Gleichwohl ist der Quotenpreistrend angesichts der weiterhin bestehenden EU-Entscheidungslage zum Auslaufen der Quote erstaunlich. Zwei Erklärungen sind möglich: Zum einen zeigen die hohen Kreditnachfragen von Milchlandwirten (Banken, AFP-Mittel), dass ein Teil der Betriebe trotz oder antizyklisch auch gerade wegen der niedrigen Erzeugerpreise investiert und Wachstumsschritte unternimmt, die sich nicht allein durch Überlieferung decken lassen. Die nachgefragte Menge überstieg das Angebot beim letzten Termin um 163000 Tonnen. Zum anderen ist zu vermuten, dass der (Zweck-) Optimismus des BDM (Bund Deutscher Milchviehhalter) hinsichtlich der Zukunftsfähigkeit der Quotenregelung die Erwartungen eines Teils der Betriebe beeinflusst. Betriebswirtschaftlich gesehen binden die expandierenden Betriebe dadurch Kapital. Eine alternative Strategie wäre, darauf zu setzen, dass entweder keine Übermengen in den nächsten Lieferjahren erreicht werden (siehe 2008/2009) oder zu hoffen, dass die überlieferte Milch 
durch eine gute Verwertung der Molkereien (siehe 2007/2008) vollständig saldiert wird.

Schließlich weist die aktuelle Sektoruntersuchung Milch des BundeSKARTELLAMTES (2009b: 68f.) darauf hin, dass ein bundesweiter Mindestpreis, wie er von Seiten des BDM mehrfach in die Diskussion gebracht wurde, gegen das Kartellverbot verstoßen würde. Auch die Milchpreisempfehlungen, die derzeit in den europäischen Nachbarländern Frankreich und Spanien ausgesprochen werden, sind nach Ansicht des Kartellamtes nicht wettbewerbskonform. Es ist daher unrealistisch von Seiten der Landwirte, auf eine solche Regelung zu hoffen, sie würde zudem nicht $\mathrm{zu}$ einer Verbesserung der Situation der Milchvieh haltenden Betriebe beitragen (BUNDESKARTELLAMT 2009b: 68f.).

\section{Die internationalen Märkte für Milchprodukte}

Nachdem im Jahr 2007 die Preisspitze auf den internationalen Märkten für Milcherzeugnisse erreicht wurde, war das Jahr 2009 durch eine Preissohle im Frühjahr, gefolgt von einem Anstieg der Preise zu Ende des Jahres gekennzeichnet. In der EU wurde marktpolitisch auf diese Entwicklung vor allem mit einer über die festgelegten Obergrenzen ausgedehnten Intervention und der Wiederaufnahme von Exporterstattungen reagiert. Ersteres dürfte sich eher stabilisierend auf die Weltmarktpreise ausgewirkt haben, während letzteres einen erneuten Druck auf die Weltmarktpreise ausübte. Trotz der Markteingriffe der EU erreichten auch die europäischen Inlandspreise historische Niedrigstände auf oder knapp unterhalb des Interventionspreisniveaus. Diese Entwicklung veranschaulicht die Abhängigkeit der Inlandspreise von den Preisentwicklungen auf den internationalen Märkten. Entsprechend bedarf es mehr denn je eines Blickes auf die internationalen Begebenheiten, um eine Aussage über die inländischen Entwicklungen treffen zu können.

\subsection{Nachfrage nach Milcherzeugnissen}

Im Jahr 2008 wurde das weltweite Nachfragewachstum deutlich vom Angebotswachstum (Kapitel 3.2) übertroffen, so dass es erstmals seit 2002 wieder zum
Aufbau beträchtlicher, zu Anfang privatwirtschaftlicher Lagerbestände (Kapitel 3.3) kam. Die Wachstumsrate der Nachfrage lag dabei mit 1,0\% deutlich unter dem Durchschnittswert der Jahre 2000 bis 2008 von 2,3\% p.a. (AMI, 2009; FAO, 2009). Für den weltweiten durchschnittlichen Pro-Kopf-Verbrauch folgte daraus ein Rückgang um $0,3 \mathrm{~kg} / \mathrm{Kopf}$ auf $101,6 \mathrm{~kg} / \mathrm{Kopf}$ (AMI, 2009).

Grundsätzlich war die Nachfrageseite in den vergangenen drei Jahren gleich von zwei wesentlichen Veränderungen der Rahmenbedingungen betroffen: Einerseits von kurzfristigen extremen Preisschwankungen, sowohl bei Milcherzeugnissen als auch bei Substituten aus dem Bereich der pflanzlichen Proteine und Öle. Andererseits zog die Wirtschafts- und Finanzkrise (WFK) einen negativen Einkommenseffekt nach sich. Beide Effekte überlagern sich in den beobachteten Änderungen auf den internationalen Märkten für Milcherzeugnisse. Werden die internationalen Handelsmengen betrachtet (Abbildung 5), so zeigt sich ein Einbruch ${ }^{1}$ des Vollmilchpulver- (VMP-) und des Butterhandels zum dritten Quartal 2007, der mit der Preisspitze des Jahres 2007 zusammenfällt. Erst zeitlich verzögert, d. h. ab dem zweiten bzw. dritten Quartal 2008, bricht der Handel mit Käse ein.

\section{Abbildung 5. Gleitender Durchschnitt der Quartals-Handels- mengen ${ }^{1)}$ auf den wichtigsten internationalen Märkten für Milcherzeugnisse}

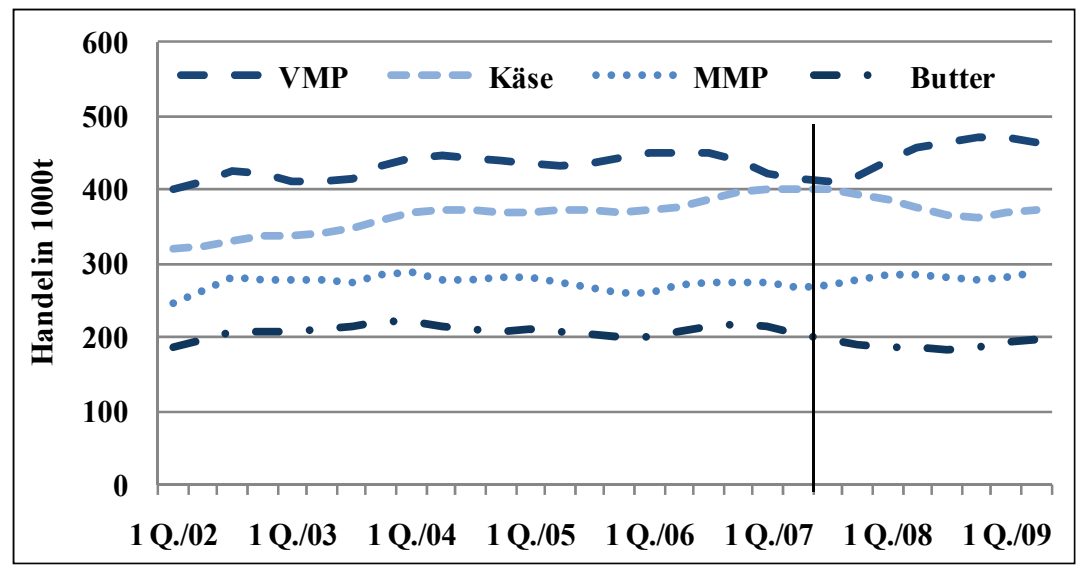

1) Die dargestellten Mengen entsprechen den Exportmengen der 79 durch die GTISDatenbank erfassten Länder. Damit sind alle wichtigen Exporteure auf den Weltmärkten für Milcherzeugnisse abgedeckt.

Quelle: GTIS (2009)
1 Dadurch, dass in Abbildung 1 ein gleitender Durchschnitt der Handelsmengen verwendet wird, ist die tatsächliche Dynamik auf den Märkten, d.h. kurzfristige Einbrüche von Handelsmengen in einzelnen Monaten bzw. Quartalen, nicht erkennbar. 
Diese zeitliche Verzögerung und das Verbleiben auf einem niedrigeren Niveau kann nicht allein mit einer zeitlich leicht versetzten Entwicklung ${ }^{2}$ der Käsepreise erklärt werden. Vielmehr ist zu vermuten, dass beim Käsehandel auch schon Auswirkungen der WFK zu sehen sind. Dies wäre durchaus zu erwarten: Zum einen findet der Hauptkonsum von Käse in den Industrieländern statt, welche besonders von der WFK betroffen sind, und zum anderen dürfte Käse als höher veredeltes Produkt auch eine höhere Einkommenselastizität aufweisen, wodurch die Käsenachfrage von einem Einkommensschock stärker beeinflusst wird. Im Gegensatz dazu ist bei den Handelsmengen von Magermilchpulver (MMP), VMP und Butter bisher kein eindeutiger Einfluss der WFK sichtbar. Speziell bei VMP ist sogar zu beobachten, dass die Handelsmengen trotz der WFK mit sinkenden Preisen wieder stark ansteigen und auch das Niveau von 2006 übertreffen.

Ein Effekt, der in Abbildung 5 nur bedingt zu erkennen ist, ist ein mögliches Wechseln internationaler Nachfrager zu preisgünstigeren Milcherzeugnissen der gleichen Kategorie infolge höherer Preise oder der WFK. Dieses Phänomen wird von DAIRY AUSTRALIA (2009a: 15) als „trading down“ auf den Weltmärkten für Milcherzeugnisse beschrieben. Ferner konstatieren die Autoren Schwierigkeiten bei Handelskrediten und -versicherungen als weitere Auswirkungen der WFK auf die internationale Nachfrage.

Für die Betrachtung der wichtigsten Importländer zeigen die Tabellen 8 und 9 die Veränderungen der Importmengen der einzelnen Milcherzeugnisse. Unmittelbar sichtbar wird bei Butter, dass ein Großteil der Länder im Jahr 2009 bedeutend weniger importierte als 2006. Die größten Auswirkungen auf die Welthandelsmenge von Butter ergeben sich dabei aus den prozentualen Veränderungen bei Russland und der EU. Im Falle der EU ist bemerkenswert, dass auch bei Käse und MMP ein signifikanter Rückgang der Importe erfolgte. Dies kam fast ausschließlich durch stark rückläufige Einfuhren aus Neuseeland zustande. Offensichtlich ist mit einem Angleichen der innereuropäischen an die internationalen Preise der euro-

2 Neben einem leichten zeitlichen Verzug der Käsepreise auf den internationalen Märkten wurde in Abbildung 1 gezeigt, dass die Endverbraucherpreise für Käse in Deutschland etwas später anzogen, aber deutlich später und nicht im gleichen Maße wieder nachgaben. Sollte dieses Phänomen auch in anderen Ländern aufgetreten sein, kann dies ebenfalls einen Grund für den Einbruch des Käsehandels darstellen. päische Markt (trotz bestehender Zollkontingente) weniger interessant für Fonterra. Somit kann der Rückgang der EU-Importe nicht als gesunkene Nachfrage interpretiert werden. Für die rückläufigen russischen Importe könnte dies jedoch eine zutreffende Erklärung sein, so verweisen DAIRY AUSTRALIA (2009a: 13, 20) auf negative Einflüsse der WFK und sinkender Ölpreise auf die russische Nachfrage nach Butter und Käse. Zwar sehen DAIRY AUSTRALIA (2009b: 7, 15) zur zweiten Jahreshälfte Anzeichen für eine Belebung der russischen Importnachfrage, dies könnte jedoch durch die von der russischen Regierung beschlossenen Importzollanhebungen bei Milch, Sahne, Butter und Säuglingsnahrungspulver konterkariert werden (USDA-FAS, 2009c; USDA-FAS 2009d). Neben dem Rückgang der russischen und europäischen Käseimporte ergeben sich die größten Auswirkungen auf die Welthandelsmenge von Käse durch die rückläufigen Importe in Japan, den USA und Saudi-Arabien. DAIRY AUSTRALIA (2009a: 13, 20) merken zudem an, dass das besonders von der WFK betroffene Japan u.a. hohe Käsebestände aufgebaut hat, was die japanischen Käseimporte bis ins Jahr 2010 dämpfen wird.

Im Gegensatz zu den Entwicklungen bei Butter und Käse sind auf dem MMP-Markt keine klaren Abwärtsbewegungen der Handelsmenge erkennbar. Im Jahr 2008 konnte Mexiko als größter MMPImporteur sogar einen beachtlichen Importzuwachs ausweisen. Dieser bestand vornehmlich aus US-Importen und setzte mit dem Jahr 2008 nach der vollen NAFTA-Implementierung in Mexiko ein (USDAFAS, 2009d). Weiterhin ist in Tabelle 9 erkennbar, dass sowohl die chinesischen MMP- als auch VMPImporte im Jahr 2007 einbrachen, was gegen die oftmals vermutete Rolle chinesischer Importe bei den Preissteigerungen des Jahres 2007 spricht. Beachtlich ist jedoch, dass China in den ersten drei Quartalen 2009 - ähnlich wie Brasilien - einen gewaltigen Anstieg der VMP-Importe aufwies. Dieser wird nicht zuletzt auch auf den chinesischen Melaminskandal zurückzuführen sein, der das Vertrauen in inländische Milcherzeugnisse untergraben hat (USDA-FAS, 2009b: 5). Werden $\mathrm{zu}$ den Importsteigerungen Chinas und Brasiliens noch die Algeriens und Venezuelas hinzugerechnet, so zeigt sich, dass diese Länder fast für den gesamten Aufwärtstrend der VMP-Handelsmengen (Abbildung 5) seit 2008 verantwortlich sind. Allerdings ist hinzuzufügen, dass die gleichen Länder auch einen bedeutenden Anteil am Einbruch der VMPHandelsmengen im Jahr 2007 hatten. Entscheidend dürfte vor allem sein, wie nachhaltig die beobachtete Steigerung der Importnachfrage ist; dies gilt auch für 
Tabelle 8. Veränderung der Importmengen der Top-10 ${ }^{1)}$ Butter- und Käse-Importländer

\begin{tabular}{|c|c|c|c|c|c|c|c|c|c|}
\hline \multirow{2}{*}{$\begin{array}{l}\text { Butter- } \\
\text { Importeure }\end{array}$} & \multirow{3}{*}{$\begin{array}{r}\begin{array}{r}\text { Markt- } \\
\text { anteil }\end{array} \\
12 \%\end{array}$} & \multicolumn{3}{|c|}{ Veränderung der Importe } & \multirow{2}{*}{$\begin{array}{l}\text { Käse- } \\
\text { Importeure }\end{array}$} & \multirow{3}{*}{$\begin{array}{r}\begin{array}{r}\text { Markt- } \\
\text { anteil }\end{array} \\
15 \%\end{array}$} & \multicolumn{3}{|c|}{ Veränderung der Importe } \\
\hline & & 2007 & 2008 & $2009^{2)}$ & & & 2007 & 2008 & $2009^{2)}$ \\
\hline 1 Russland & & $-33 \%$ & $12 \%$ & $-32 \%$ & 1 Japan & & $9 \%$ & $-17 \%$ & $-4 \%$ \\
\hline 2 EU-27 & $11 \%$ & $1 \%$ & $-30 \%$ & $-31 \%$ & 2 USA & $15 \%$ & $-2 \%$ & $-15 \%$ & $2 \%$ \\
\hline 3 Ägypten & $7 \%$ & $12 \%$ & $-7 \%$ & $70 \%$ & 3 Russland & $12 \%$ & $7 \%$ & $6 \%$ & $-10 \%$ \\
\hline 4 Mexiko & $6 \%$ & $21 \%$ & $-31 \%$ & $41 \%$ & 4 EU-27 & $8 \%$ & $-6 \%$ & $-10 \%$ & $-1 \%$ \\
\hline 5 USA & $5 \%$ & $1 \%$ & $-55 \%$ & $220 \%$ & 5 Mexiko & $5 \%$ & $10 \%$ & $-21 \%$ & $2 \%$ \\
\hline 6 Iran & $5 \%$ & $14 \%$ & $29 \%$ & $5 \%$ & 6 Saudi-Arabien & $5 \%$ & $-4 \%$ & $-22 \%$ & $-14 \%$ \\
\hline 7 Saudi-Arabien & $5 \%$ & $-10 \%$ & $2 \%$ & $-3 \%$ & 7 Australien & $4 \%$ & $1 \%$ & $5 \%$ & $-15 \%$ \\
\hline 8 Marokko & $4 \%$ & $-35 \%$ & $46 \%$ & $-20 \%$ & 8 Südkorea & $3 \%$ & $12 \%$ & $-4 \%$ & $1 \%$ \\
\hline 9 Singapur & $3 \%$ & $6 \%$ & $-17 \%$ & $-23 \%$ & 9 Schweiz & $2 \%$ & $12 \%$ & $10 \%$ & $7 \%$ \\
\hline 10 Kanada & $2 \%$ & $-10 \%$ & $-44 \%$ & $72 \%$ & 10 Kanada & $2 \%$ & $3 \%$ & $0 \%$ & $-13 \%$ \\
\hline
\end{tabular}

${ }^{1)}$ Der Marktanteil wurde nach der gesamten Importmenge der Länder im Zeitraum 1999 bis 2008 bestimmt.

${ }^{2)}$ Die Veränderung der Importe im Jahr 2009 berücksichtigt nur die ersten drei Quartale im Vergleich zum Vorjahreszeitraum. Quelle: eigene Berechnungen nach Daten von GTIS (2009)

Tabelle 9. Veränderung der Importmengen der Top-10 ${ }^{1)}$ MMP- und VMP-Importländer

\begin{tabular}{|c|c|c|c|c|c|c|c|c|c|}
\hline \multirow{2}{*}{$\begin{array}{l}\text { MMP- } \\
\text { Importeure }\end{array}$} & \multirow{3}{*}{$\begin{array}{r}\text { Markt- } \\
\text { anteil } \\
12 \%\end{array}$} & \multicolumn{3}{|c|}{ Veränderung der Importe } & \multirow{2}{*}{$\begin{array}{l}\text { VMP- } \\
\text { Importeure }\end{array}$} & \multirow{3}{*}{$\begin{array}{r}\text { Markt- } \\
\text { anteil } \\
8 \%\end{array}$} & \multicolumn{3}{|c|}{ Veränderung der Importe } \\
\hline & & 2007 & 2008 & $2009^{2)}$ & & & 2007 & 2008 & $2009^{2)}$ \\
\hline 1 Mexiko & & $9 \%$ & $26 \%$ & $-1 \%$ & 1 Algerien & & $-12 \%$ & $-4 \%$ & $41 \%$ \\
\hline 2 Philippinen & $9 \%$ & $-2 \%$ & $-14 \%$ & $-1 \%$ & 2 Saudi-Arabien & $5 \%$ & $-4 \%$ & $-7 \%$ & $-23 \%$ \\
\hline 3 Algerien & $8 \%$ & $34 \%$ & $15 \%$ & $8 \%$ & 3 Venezuela & $4 \%$ & $3 \%$ & $151 \%$ & $0 \%$ \\
\hline 4 Indonesien & $7 \%$ & $5 \%$ & $-15 \%$ & $21 \%$ & 4 China & $4 \%$ & $-24 \%$ & $-14 \%$ & $529 \%$ \\
\hline 5 Malaysia & $6 \%$ & $41 \%$ & $-7 \%$ & $-1 \%$ & 5 Brasilien & $3 \%$ & $-39 \%$ & $20 \%$ & $221 \%$ \\
\hline 6 Thailand & $6 \%$ & $-15 \%$ & $7 \%$ & $-27 \%$ & 6 Nigeria & $3 \%$ & $-6 \%$ & $2 \%$ & $9 \%$ \\
\hline 7 Singapur & $5 \%$ & $4 \%$ & $-13 \%$ & $6 \%$ & 7 Sri Lanka & $3 \%$ & $-5 \%$ & $7 \%$ & $-6 \%$ \\
\hline 8 Japan & $4 \%$ & $11 \%$ & $-9 \%$ & $21 \%$ & 8 Oman & $3 \%$ & $-10 \%$ & $80 \%$ & $4 \%$ \\
\hline 9 China & $4 \%$ & $-35 \%$ & $36 \%$ & $12 \%$ & 9 Philippinen & $3 \%$ & $-13 \%$ & $11 \%$ & $-20 \%$ \\
\hline 10 EU-27 & $3 \%$ & $-54 \%$ & $-22 \%$ & $-27 \%$ & 10 Mexiko & $2 \%$ & $6 \%$ & $-49 \%$ & $14 \%$ \\
\hline
\end{tabular}

${ }^{1)}$ Der Marktanteil wurde nach der gesamten Importmenge der Länder im Zeitraum 1999 bis 2008 bestimmt.

${ }^{2)}$ Die Veränderung der Importe im Jahr 2009 berücksichtigt nur die ersten drei Quartale (bzw. zwei Quartale im Falle Algeriens) im Vergleich zum Vorjahreszeitraum.

Quelle: eigene Berechnungen nach Daten von GTIS (2009)

leicht positive Tendenzen beim Butterhandel im zweiten und dritten Quartal 2009. DAIRY AUSTRALIA (2009b: 15) verweisen darauf, dass es sich bei der vermeintlich beobachteten Nachfrage auch um strategische Käufe handeln könnte, um einem potentiell begrenzten Angebot Anfang 2010 entgegenzutreten. Mit anderen Worten, es könnten erneut spekulative Lagerbestände angelegt werden oder aufgrund sinkender Preise sehr niedrige Lagerbestände wieder aufgefüllt werden. Zumindest im Falle von Mexiko und Algerien dürfte jedoch eine Reihe von Markteingriffen der Regierungen die Importmengen stabilisieren (DAIRY AUSTRALIA, 2009b: 15; USDA-FAS, 2009e).

\subsection{Angebot von Milcherzeugnissen}

Das Angebot ist in Form der weltweiten Milchproduktion im Jahr 2008 mit 1,6\% deutlich stärker gewachsen als die Nachfrage. Dennoch liegt dieser Wert noch unter dem Durchschnitt des Zeitraums 2000 bis 2008, in welchem die Milchproduktion mit 2,3\% p.a. wuchs (AMI, 2009; FAO, 2009).

Ähnlich wie die Nachfrage war auch das Angebot in den vergangenen Jahren von verschiedenen Änderungen der Rahmenbedingungen betroffen. Hierunter fallen zum einen extrem unterschiedliche Milchpreisniveaus innerhalb von kürzester Zeit sowie ein 
Anstieg - und mittlerweile auch wieder ein Rückgang - der Futter- bzw. allgemein der Inputkosten. Auch wenn letztere im Jahr 2009 rückläufig waren, stießen doch oftmals hohe Inputkosten auf geringe Milchpreise, was sich negativ auf das Milchproduktionswachstum auswirkte. So nahm bspw. in der EU-27 trotz einer Quotenanhebung von einem Prozent für das Quotenjahr 2009/10 die Produktion in den ersten zehn Monaten 2009 um 0,4 \% gegenüber dem Vorjahr $\mathrm{ab}$ (EUROSTAT, 2009). Innerhalb der EU konnten allerdings auch 9 Länder ihre Produktion steigern. An der Spitze dieser Länder stehen Belgien $(+5,0 \%)$, Dänemark (+3,5\%) und Deutschland $(+3,3 \%)$. Auf der anderen Seite nahm die Produktion in 17 Ländern ab. Die von der Gesamtmenge bedeutendsten Rückgänge verzeichneten dabei Frankreich $(-4,4 \%)$, Irland $(-2,7 \%)$ und Großbritannien $(-1,0 \%)$ (ebenda).

Im Außenhandel waren die vergangenen Jahre von Verlusten an Exportmengen für die EU geprägt (Tabelle 10 und 11). Dies war am deutlichsten bei Butter ausgebildet. So brachen die europäischen Butterexporte ab Mitte 2007 ein und haben sich seit- dem noch nicht wieder erholt. Dies ist nur zu einem geringen Teil mit den im vorigen Kapitel konstatierten geringeren Importen zu erklären. Auch die Wiedereinführung von Exporterstattungen zum 24. Januar 2009 hat an dieser Situation nichts geändert. Interessant ist in diesem Zusammenhang, dass die Exportpreise der EU während der Zahlung von Exporterstattungen im Jahr 2009 nur unwesentlich geringer waren als die Inlandspreise der EU (Abbildung 8). Die Exporterstattungen wurden also offensichtlich größtenteils nicht in den Preisverhandlungen mit internationalen Nachfragern berücksichtigt und trugen somit nicht zu einem wettbewerbsfähigen Preis der europäischen Exporte bei. $\mathrm{Zu}$ diesen Überlegungen passt die Beobachtung, dass die Butterexportpreise der EU bereits seit der Preishausse im Jahr 2007 durchschnittlich $28 \%$ über denen Ozeaniens liegen; normalerweise existieren hier nur geringe Differenzen. Nennenswerte Anstiege der Exporte konnte die EU lediglich bei MMP im Jahr 2007 und bei VMP im Jahr 2008 ausweisen. Bei MMP erfolgte dieser Anstieg als Reaktion auf die Preishausse, wobei ein starker Einbruch der EU-MMP-Exporte im Jahre 2006 vorausging. Mit

Tabelle 10. Veränderung der Exportmengen der Top- $7^{1)}$ Butter- und Käse-Exportländer

\begin{tabular}{|c|c|c|c|c|c|c|c|c|c|}
\hline \multirow{2}{*}{$\begin{array}{l}\text { Butter- } \\
\text { Exporteure }\end{array}$} & \multirow{2}{*}{$\begin{array}{r}\text { Markt- } \\
\text { anteil }\end{array}$} & \multicolumn{3}{|c|}{ Veränderung der Exporte } & \multirow{2}{*}{$\begin{array}{l}\text { Käse- } \\
\text { Exporteure }\end{array}$} & \multirow{2}{*}{$\begin{array}{r}\text { Markt- } \\
\text { anteil }\end{array}$} & \multicolumn{3}{|c|}{ Veränderung der Exporte } \\
\hline & & 2007 & 2008 & $2009^{2)}$ & & & 2007 & 2008 & $2009^{2)}$ \\
\hline 1 Neuseeland & $48 \%$ & $-7 \%$ & $-10 \%$ & $39 \%$ & 1 EU-27 & $39 \%$ & $2 \%$ & $-7 \%$ & $1 \%$ \\
\hline 2 EU-27 & $29 \%$ & $-12 \%$ & $-28 \%$ & $3 \%$ & 2 Neuseeland & $21 \%$ & $4 \%$ & $-20 \%$ & $19 \%$ \\
\hline 3 Australien & $11 \%$ & $-16 \%$ & $-27 \%$ & $92 \%$ & 3 Australien & $15 \%$ & $4 \%$ & $-27 \%$ & $-2 \%$ \\
\hline 4 USA & $3 \%$ & $258 \%$ & $113 \%$ & $-81 \%$ & 4 USA & $5 \%$ & $40 \%$ & $32 \%$ & $-26 \%$ \\
\hline 5 Ukraine & $2 \%$ & $-69 \%$ & $56 \%$ & $-89 \%$ & 5 Ukraine & $5 \%$ & $26 \%$ & $25 \%$ & $-2 \%$ \\
\hline 6 Kanada & $2 \%$ & $-27 \%$ & $-89 \%$ & $107 \%$ & 6 Schweiz & $4 \%$ & $7 \%$ & $3 \%$ & $2 \%$ \\
\hline 7 Argentinien & $1 \%$ & $19 \%$ & $18 \%$ & $-34 \%$ & 7 Argentinien & $3 \%$ & $-22 \%$ & $-20 \%$ & $23 \%$ \\
\hline
\end{tabular}

${ }^{1)}$ Der Marktanteil wurde nach der gesamten Exportmenge der Länder im Zeitraum 1999 bis 2008 bestimmt.

${ }^{2)}$ Die Veränderung der Exporte im Jahr 2009 berücksichtigt nur die ersten drei Quartale im Vergleich zum Vorjahreszeitraum.

Quelle: eigene Berechnungen nach Daten von GTIS (2009)

Tabelle 11. Veränderung der Exportmengen der Top- $7^{1)}$ MMP- und VMP-Exportländer

\begin{tabular}{|c|c|c|c|c|c|c|c|c|c|}
\hline \multirow{2}{*}{$\begin{array}{l}\text { MMP- } \\
\text { Exporteure }\end{array}$} & \multirow{2}{*}{$\begin{array}{r}\text { Markt- } \\
\text { anteil }\end{array}$} & \multicolumn{3}{|c|}{ Veränderung der Exporte } & \multirow{2}{*}{$\begin{array}{l}\text { VMP- } \\
\text { Exporteure }\end{array}$} & \multirow{2}{*}{$\begin{array}{r}\text { Markt- } \\
\text { anteil }\end{array}$} & \multicolumn{3}{|c|}{ Veränderung der Exporte } \\
\hline & & 2007 & 2008 & $2009^{2)}$ & & & 2007 & 2008 & $2009^{2)}$ \\
\hline 1 Neuseeland & $29 \%$ & $-11 \%$ & $-14 \%$ & $69 \%$ & 1 Neuseeland & $37 \%$ & $5 \%$ & $-13 \%$ & $39 \%$ \\
\hline 2 EU-27 & $24 \%$ & $140 \%$ & $-12 \%$ & $9 \%$ & 2 EU-27 & $33 \%$ & $-16 \%$ & $32 \%$ & $-4 \%$ \\
\hline 3 Australien & $23 \%$ & $-28 \%$ & $-6 \%$ & $55 \%$ & 3 Australien & $9 \%$ & $-32 \%$ & $32 \%$ & $20 \%$ \\
\hline 4 USA & $21 \%$ & $-11 \%$ & $51 \%$ & $-44 \%$ & 4 Argentinien & $9 \%$ & $-52 \%$ & $2 \%$ & $82 \%$ \\
\hline 5 Ukraine & $5 \%$ & $-10 \%$ & $-24 \%$ & $-38 \%$ & 5 Uruguay & $2 \%$ & $-14 \%$ & $16 \%$ & $33 \%$ \\
\hline 6 Kanada & $4 \%$ & $5 \%$ & $-23 \%$ & $-10 \%$ & 6 Brasilien & $1 \%$ & $137 \%$ & $98 \%$ & $-82 \%$ \\
\hline 7 Argentinien & $2 \%$ & $-53 \%$ & $31 \%$ & $-4 \%$ & 7 USA & $1 \%$ & $166 \%$ & $80 \%$ & $-58 \%$ \\
\hline
\end{tabular}

${ }^{1)}$ Der Marktanteil wurde nach der gesamten Exportmenge der Länder im Zeitraum 1999 bis 2008 bestimmt.

${ }^{2)}$ Die Veränderung der Exporte im Jahr 2009 berücksichtigt nur die ersten drei Quartale im Vergleich zum Vorjahreszeitraum.

Quelle: eigene Berechnungen nach Daten von GTIS (2009) 
dem Anstieg der VMP-Exporte konnte die EU im Jahr 2008 nach vorherigen Einbrüchen vom Wachstum des VMPMarktes profitieren, was sich jedoch in den ersten drei Quartalen 2009 nicht fortsetzen ließ.

Für die USA zeigt Abbildung 6 einen Einbruch der Milchproduktion, welcher einer Reihe von Jahren kontinuierlichen Wachstums folgt. Ein Einflussfaktor für den Rückgang der USMilcherzeugung dürfte die Kombination von hohen Futterkosten und geringen Milchpreisen sein. Als Maßstab dafür kann das „Milk-Feed Price Ratio“"3 in den USA herangezogen werden. Dieser Wert erreichte im Juni 2009 einen Tiefstand von 1,45 (Gould, 2009). Wie dramatisch niedrig dieser Wert ist, zeigt ein Vergleich mit der Entwicklung der Jahre 1985 bis 2008. In dieser Zeit lag das Milk-Feed Price Ratio durchschnittlich bei 2,9 mit den Extremwerten 2,1 und 4,3 (ebenda). Ein weiterer und unmittelbar wirksamer Einflussfaktor für die geringe Milchproduktion in den USA sind die Abschlachtprogramme des privatwirtschaftlich organisierten „Cooperatives Working Together" (CWT). Seit Juni 2008 wurden dabei insgesamt fünf Runden durchgeführt, wobei in den ersten vier 250368 Kühe und 5291 Färsen geschlachtet wurden. Hinzu kommen 26412 Kühe und 465 Färsen, die in der letzten bzw. dritten Abschlachtrunde des Jahres 2009 gemeldet wurden (CWT, 2009; NMPF, 2009). USDA-FAS (2009b: 3) berichten, dass damit die Kuhzahl in den USA um insgesamt 2,4 \% reduziert wurde und erwarten für 2010 einen Rückgang der Milchproduktion um bis zu ein Prozent.

Nachdem die USA in den Jahren 2007 und/oder 2008 den Export von Butter, Käse, MMP und VMP beträchtlich ausdehnen konnten, brach die Ausfuhr dieser Produkte in den ersten drei Quartalen 2009 wieder ein (Tabelle 10 und 11). Am bedeutendsten für die Weltmärkte war dies bei MMP und Butter ausgeprägt und damit bei den Produkten, für die staatliche Aufkauf- bzw. Interventionsprogramme in den USA existieren. Damit wurde der Preisdruck auf den Weltmärkten deutlich entschärft. Andererseits kündigte die US-Regierung im Mai 2009 auch eine Wiederauf-

3 Das Milk-Feed Price Ratio ist das Preisverhältnis zwischen einem Kilogramm Milch und einem Kilogramm Futter und ein Maßstab für die Profitabilität der Milchproduktion. nahme von Exporterstattungen über das „Dairy Export Incentive Program“ (DEIP) vom 1. Juli 2009 bis 30. Juni 2010 an. Die Obergrenzen, die die USA nach den WTO-Vereinbarungen dabei einhalten müssen, betragen $68201 \mathrm{t}$ MMP, $21097 \mathrm{t}$ Butterfett und $3030 \mathrm{t}$ verschiedener Käsesorten (DAIRY AUSTRALIA, 2009a; USDA-FAS, 2009f).

Neuseeland konnte im Jahr 2009 die Milchproduktion wieder deutlich ausdehnen, nachdem im Vorjahr ein dürrebedingter Rückgang der Produktion zu verzeichnen war (Abbildung 6). Auch haben die Rekordmilchpreise des Jahres 2008 dazu geführt, dass in Neuseeland bis Ende 2008330 neue Milchproduzenten mit 165000 Kühen die Produktion aufgenommen haben (USDA-FAS, 2009a: 4). So konnten die neuseeländischen Exporte von Butter, MMP und VMP in den ersten drei Quartalen 2009 beträchtlich ausgedehnt werden (Tabelle 10 und 11). Die inländische Milchproduktionssteigerung und ein leichter Rückgang beim Export anderer Milcherzeugnisse (wie z.B. Käse) reichen jedoch nicht aus, um die beobachtete Exportmengensteigerung zu erklären. Dies deutet darauf hin, dass ab Anfang 2009 ein großer Teil von zuvor aufgebauten neuseeländischen Lagerbeständen geräumt wurde. Obwohl die Milchproduktion in Neuseeland zu Beginn der aktuellen Saison 2009/10 leicht rückläufig war, gehen USDA-FAS (2009b: 3) davon aus, dass in der gesamten Saison eine Steigerung von 2,5\% zu verzeichnen sein wird.

Australien verbuchte 2009 erstmals seit 2005 wieder ein Wachstum der Milchproduktion (Abbildung 6), wenngleich das bisherige Höchstniveau von 2002 noch nicht wieder erreicht wurde. Vor allem 
in den ersten drei Quartalen 2009 konnten damit nach den Exportrückgängen der Vorjahre wieder teilweise hohe Zuwächse bei Butter, MMP und VMP erreicht werden (Tabelle 10 und 11). In der aktuellen Saison ist für Australien jedoch bereits ein erneuter Rückgang der Milchproduktion abzusehen, der sich in einen negativen Gesamttrend seit 2002 einreiht. USDA-FAS (2009b: 3) gehen von einem Rückgang von $1,0 \%$ in der aktuellen Saison 2009/10 aus, der vor allem durch eine geringere Kuhzahl bedingt ist. Dies lässt Zweifel daran aufkommen, dass Australien mittel- und auch langfristig wieder an die deutlich höheren Weltmarktanteile des Jahres 2002 anschließen kann. In diesem $\mathrm{Zu}-$ sammenhang sei darauf verwiesen, dass dem Rückgang der Milchproduktion eine fast vollständige Deregulierung der australischen Milcherzeugung im Jahr 2000 vorausging (ARMENTANO et al., 2004: 49), die die relative Vorzüglichkeit der Milchproduktion beeinträchtigt haben könnte.

In Südamerika konnte Argentinien nach einem starken Produktionseinbruch im Jahr 2007 nun im zweiten Jahr in Folge einen Produktionszuwachs verzeichnen (Abbildung 6). Dieser äußerte sich im Jahr 2008 vornehmlich in steigenden Butter- und MMPExporten, wohingegen in den ersten drei Quartalen 2009 vor allem die Käse- und VMP-Exporte anstiegen. Brasilien hat sich mittlerweile vom Nettoimporteur durch eine Steigerung der Milchproduktion von $6 \%$ p.a. zwischen 2006 und 2008 zu einem Exportland entwickelt und erlangt speziell als VMP-Exporteur eine stärkere Bedeutung (USDA-FAS, 2009a: 3) (Tabelle 11). Diese Entwicklung erfuhr allerdings in 2009 - u.a. aufgrund eines Einbruchs der Milchproduktion um voraussichtlich $4 \%$ (DAIRY AUSTRALIA, 2009b: 19) - einen Rückschlag. Für die Ukraine war das Jahr 2009 das fünfte in Folge mit einem starken Rückgang der Produktion. Darunter litten vor allem die Exporte von MMP und Butter, womit die Bedeutung der Ukraine auf diesen Märkten stark zurückgeht.

Zusammenfassend war die Angebotssituation im Jahr 2009 von mehr oder weniger stark sinkenden Exporten der USA und EU gekennzeichnet, die ausgeglichen wurden von zum Teil beachtlich steigenden Exporten aus Ozeanien und Südamerika. Damit haben sich zumindest in 2009 die Weltmarktanteile deutlich verschoben.

\subsection{Entwicklung der Lagerbestände}

Nachdem die Lagerbestände im Jahr 2007 auf historischen Niedrigständen angekommen waren, konnten bereits Mitte 2008 Anzeichen für wachsende private Lagerbestände gesehen werden. Diese wurden in der Folge größtenteils in öffentliche Lagerbestände überführt. In den USA existiert hierzu die „Commodity Credit Corporation“ (CCC), über die MMP angekauft wird, ergänzt durch ein kleineres Programm zum Ankauf von Butter (DAIRY AUSTRALIA 2009a: 16). Die Ankäufe der CCC begannen im Oktober 2008 (USDA-FSA, 2008), während die Interventionsstellen in der EU erst im März 2009 öffneten. Binnen weniger Monate wurden über die Intervention große Mengen MMP und Butter vom Markt genommen (Abbildung 7). Die Obergrenzen für die obligatorische Intervention in der EU (30 $000 \mathrm{t}$ Butter / $109000 \mathrm{t}$ MMP) wurden dabei deutlich überschritten. ${ }^{4}$ Ferner wurde die Intervention im Rahmen von Ausschreibungsverfahren über den August hinaus bis zum 28. Februar 2010 bzw. wenn erforderlich sogar bis zum 28. Februar 2011 verlängert; ebenso wurden die Beihilfen für die private Lagerhaltung verlängert (EU-KOMMISSION, 2009a: 6, 7; EU-AMTSBLATT, 2009).

Im dritten Quartal 2009 erreichten die so zustande gekommenen Lagerbestände ihren vorläufigen Höchstwert (Abbildung 7). In der Spitze lagen damit $38 \%$ des jährlichen Welt-MMP-Handels und $39 \%$ des jähr-

4 Bis Ende Oktober 2009 gingen $76646 \mathrm{t}$ Butter und 267740 t MMP in die Intervention. 
lichen Welt-Butter-Handels in europäischen und USamerikanischen Lagern. Der Einfluss dieser Bestände auf die Entwicklung der Märkte wird davon abhängen, in welcher Form ein Bestandsabbau durchgeführt wird. In den USA soll ein Großteil der Bestände (93 000 t MMP) für inländische Hilfsprogramme verwendet werden, ergänzt durch geringere Mengen für internationale Nahrungsmittelhilfe (DAIRY AUSTRALIA 2009a: 16). Für die EU-Interventionsbestände gilt, dass diese solange eingelagert bleiben sollen, „bis sie auf dem Binnenmarkt oder dem Weltmarkt ohne Störungen abgesetzt werden können“" (EU-KOMMISSION, 2009a: 7). Dabei soll auch in der EU teilweise auf Hilfsprogramme gesetzt werden. So wurde für 2010 ein Nahrungsmittelhilfeprogramm verabschiedet, an dem 19 Mitgliedsstaaten teilnehmen und für das 65290 t MMP sowie 51148 t Butter aus Interventionsbeständen verwendet werden (EU-KOMMISSION, 2009b). Trotz der Nutzung für Hilfsprogramme ist zu befürchten, dass diese Mengen teilweise auch zur Verdrängung von Marktnachfrage führen. Ferner ist zu beachten, dass auch noch private Lagerbestände existieren, deren Höhe schwer abzuschätzen ist.

\subsection{Preisentwicklung}

In Abbildung 8 sind die Preisentwicklungen der letzten Jahre anhand des aus Butter und MMP abgeleiteten Milchrohstoffwertes (MRW) dargestellt. Dabei wird vor allem der starke Absturz der Preise nach der Preishausse des Jahres 2007 sichtbar. Dieser Absturz kann als unmittelbare Folge der Angebots- und Nachfragereaktionen der Preishausse gesehen werden und wurde im Jahr 2008 lediglich durch ein negatives Produktionswachstum (bzw. dessen Prognose) in Neuseeland verzögert (USDA-FAS, 2009a). Ein direkter Einfluss der WFK auf den Preisabsturz ist hingegen unwahrscheinlich, da der Preisrutsch im Jahr 2008 bereits im August erfolgte und das Resultat steigender privatwirtschaftlicher Lagerbestände, also eines Ungleichgewichtes im Markt, war. Grundsätzlich dürften auch psychologideutlichen Abweichungen führt. sche Faktoren den Preisabsturz verstärkt haben. Das heißt, in Erwartung sinkender Preise wurde nur der kurzfristige Bedarf nachgefragt und Lagerbestände möglichst abgebaut. Ein solches Verhalten setzt die Preise noch stärker unter Druck. Ein Bereich, bei dem die WFK hingegen durchaus einen Einfluss auf die Preise haben kann, ist die Tiefe der Preissohle in 2009 und Zeitpunkt und Geschwindigkeit des erneuten Preisanstiegs.

Ein wichtiger Einflussfaktor, weswegen die sinkenden Preise Anfang 2009 auf dem beobachteten Niveau in eine Preissohle mündeten, waren die Interventionsaktivitäten der USA und der EU. Das heißt, durch den massiven staatlichen Aufkauf von MMP und Butter sanken die Exporte beider Länder und die Preise wurden stabilisiert.

Die Wiederaufnahme von Exporterstattungen wirkte hingegen in die entgegengesetzte Richtung, da sie es den Exporteuren ermöglichen, zu einem um die Erstattung verringerten Preis am Weltmarkt anzubieten. Somit müssten sich der MRW (Milchrohstoffwert) in Deutschland und der virtuelle Weltmilchpreis in Abbildung 8 während der Zahlung von Exporterstattungen stärker voneinander entfernen wie das bspw. bis zum Jahr 2007 der Fall war. Dass dies 2009 nicht passierte, zeigt, dass die Exporterstattungen nicht oder kaum in den Preisverhandlungen berücksichtigt wurden (vgl. Kapitel 3.2). Der MRW in Deutschland lag hingegen ziemlich genau auf dem durch die Intervention abgesicherten Niveau.

\section{Abbildung 8. Entwicklung von MRW ${ }^{1)}$ und Milchpreis}

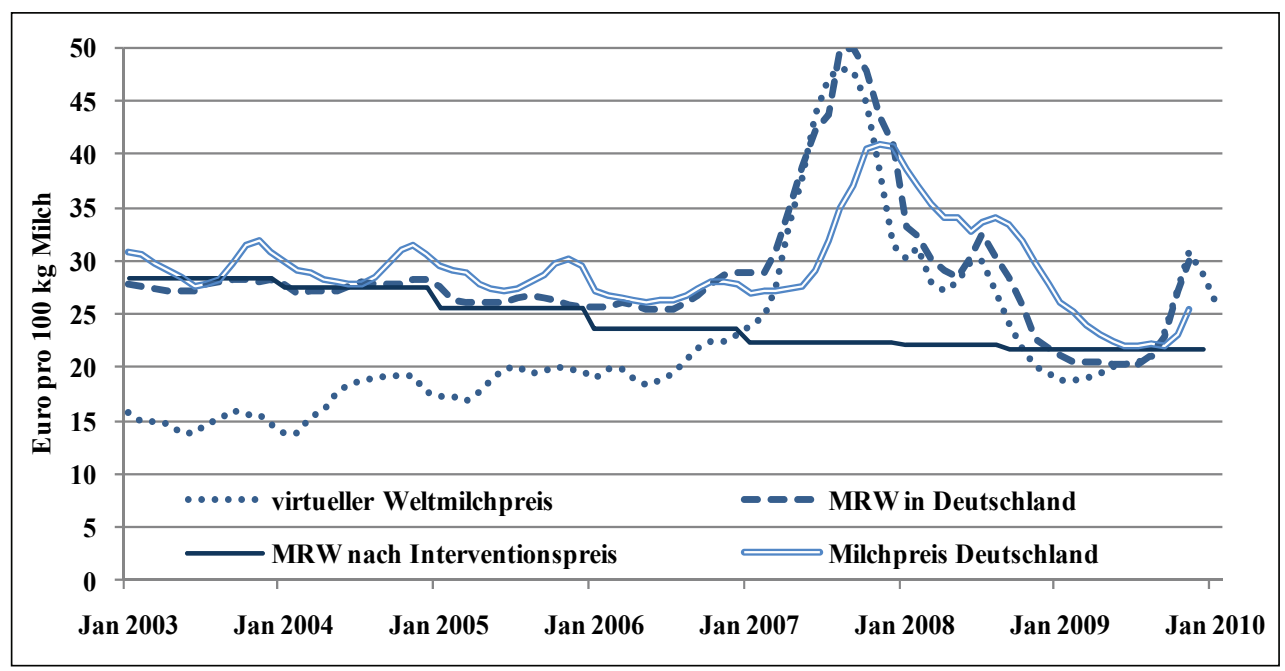

1) Der Milchrohstoffwert (MRW) setzt sich aus den Preisen für Butter und MMP (Fett- und Eiweißkomponente der Milch), abzüglich Verarbeitungskosten zusammen. Der MRW zu Exportpreisen Westeuropas wird folgend als „virtueller Weltmilchpreis“ bezeichnet. In der Literatur werden hierbei oftmals die Exportpreise Ozeaniens benutzt, was aber (abgesehen von den letzten Jahren) zu keinen

Quellen: USDA-AMS (2010); AMI (2009); EU-KOMMISSION (2009c) 
Dass sich in Abbildung 8 dennoch Differenzen ${ }^{5}$ zwischen MRW in Deutschland und nach Intervention ergeben, ist damit zu erklären, dass der Ankauf von Butter zu $90 \%$ des Interventionspreises erfolgt (EUAMTSBLATT, 1999).

Nachdem sich die internationalen Preise ab Frühjahr 2009 auf ein konstant niedriges Niveau eingependelt hatten, waren im August und September erste deutliche Zeichen für steigende Preise zu erkennen. Der darauf folgende Preisanstieg erfolgte besonders ausgeprägt bei Butter und sorgte dafür, dass die EUKommission zum 22. Oktober (MMP), 5. November (VMP, Käse) und 19. November (Butter) die Exporterstattungen für alle Milcherzeugnisse aussetzen konnte (EU-KOMMISSION, 2009c). Auch könnte der aktuelle Preisanstieg auf eine zyklische Bewegung der Preise auf den Weltmärkten für Milcherzeugnisse hindeuten, die durch den Preisschock des Jahres 2007 induziert wurde. Solche Zyklen können bspw. durch das sogenannte „Cobweb-Theorem“ (Schweinezyklus, HANAU, 1927) erklärt werden. In den meisten Erklärungsansätzen $\mathrm{zu}$ zyklischen Preisbewegungen wirken Lagerbestände stabilisierend auf die Preisschwankungen. Trotz beträchtlicher Lagerbestände kam es jedoch im zweiten Halbjahr 2009 zu den beobachteten Preissteigerungen. Eine Erklärung dafür ist, dass die Lagerbestände im Fall der EU schlicht nicht verfügbar sind, da sich die EU-Kommission festgelegt hat, die Interventionsbestände nur „marktschonend" abzubauen und vorerst nicht freigibt. Zwar verfügen auch die USA über Interventionsbestände, diese bestehen aber fast ausschließlich aus MMP, wovon ein Großteil in Hilfsprogramme fließt (Kapitel 3.3). Noch hinzu kommen, wie bereits erwähnt, schrumpfende neuseeländische Lagerbestände. Wird von politischer Seite eine Reduzierung der Preisvolatilität angestrebt, so wäre es deutlich sinnvoller den Abbau der Interventionsbestände transparenter $\mathrm{zu}$ gestalten und bspw. zu einem festgelegten Preis zu

5

Hieraus wird nicht klar, warum trotz der Entwicklung des MRW in Deutschland Milchauszahlungspreise von 17 Euro/100 kg Milch zu beobachten waren. Als Erklärung hierfür konstatieren DAIRY AUSTRALIA (2009a: 17), dass zum Teil (ältere) privatwirtschaftliche Bestände vorhanden waren, die weder interventionsfähig waren, noch mit Exporterstattungen exportiert werden konnten. So sehen die Interventionsrichtlinien u.a. vor, dass die betreffende Ware nicht älter als 30 (MMP) bzw. 23 Tage (Butter) sein darf und mindestens 2 Tage vor der Produktion angemeldet werden muss (BLE, 2009a, 2009b). Somit könnten ältere Lagerbestände tatsächlich eine Ursache für kurzfristig niedrigere Milchauszahlungspreise sein. verkaufen, der um eine bestimmte Spanne über dem Interventionspreis liegt.

Ungewiss ist in der jetzigen Situation, wie nachhaltig die Preisanstiege von Ende 2009 sind. Für eine positive Preisentwicklung spricht dabei, dass die Milchproduktion der vier größten Exportländer - mit Ausnahme von Neuseeland - derzeitig rückläufig ist, wobei Neuseeland die Exportzuwächse der ersten drei Quartale, die vermutlich auch teilweise aus Lagerbeständen kamen, nicht komplett wird halten können. Wird davon ausgegangen, dass die neuseeländischen Lagerbestände damit überschaubar sind, so gilt dies auch für die Butterbestände insgesamt. Zwar verfügt die EU noch über größere Butterbestände aus der Intervention, davon wird allerdings ein Großteil in das europäische Nahrungsmittelhilfeprogramm fließen. Problematischer könnten hingegen die EU- und USMMP-Bestände sein. Neben diesen steht einer weiterhin positiven Preisentwicklung vor allem entgegen, dass die aktuellen Preise in USD mittlerweile wieder auf einem historisch sehr hohen Niveau sind und die beobachtete Erholung der Nachfrage ausbremsen könnte. Dies geben auch USDA-FAS (2009b: 2) zu bedenken, die aber davon ausgehen, dass eine eventuelle Preiskorrektur nach unten, die sich bereits Ende 2009 ankündigt hat (Abbildung 8), moderat ausfallen sollte.

Allerdings haben sich auch langfristig einige Rahmenbedingungen geändert. So sind sowohl die Preise für pflanzliche Substitute für Milcherzeugnisse als auch die Futterkosten gesunken. Beide Entwicklungen setzen die oberen Grenzen für einen langfristig gleichgewichtigen Milchpreis herunter. Eine positive Nachricht ist jedoch, dass die Preise des Jahres 2009 offensichtlich zu niedrig waren, um ein stabiles Gleichgewicht zu repräsentieren.

\section{Literatur}

AgrarzeItung (10.12.2009): Friesland Campina schließt Standorte. URL: http:/www.agrarzeitung.de/nachrichten/ pages/, Abrufdatum: 10.12.2009.

AltmanN, T. (2009): Berglandmilch greift gegen Überlieferer durch. In: Top Agrar 08/2009: R6-R7. Landwirtschaftsverlag, Münster.

AMI (Agrarmarkt Informations-Gesellschaft mbH) (2009): Online-Monitor Milchwirtschaft. URL: http://www.markt undpreis.de/milchwirtschaft/produktlinie/infografikenW 3D_ami_W261.asp, Abrufdatum: 16.11.2009. Bonn.

Armentano, L., W.D. Dobson, E.V. Jesse and N. Olson (2004): The Dairy Sectors of New Zealand and Australia: A Regional Study. Babcock Institute Discussion Paper No. 2004-3. University of Wisconsin, Madison, Wisconsin. 
BArRos, P.P., B. DuArTe and D. DE LuCEnA (2006): Mergers in the food retailing sector: an empirical investigation. In: European Economic Review 50 (2): 447-468.

BLE (Bundesanstalt für Landwirtschaft und Ernährung) (2009a): Richtlinien zur Durchführung des Ankaufs im Rahmen der Intervention von Butter vom 16. Februar 2009. Referat 313, BLE, Bonn.

- (2009b): Richtlinien zur Durchführung des Ankaufs im Rahmen der Intervention von Sprühmagermilchpulver vom 20. Februar 2009. Referat 313, BLE, Bonn.

BMELV (Bundesministerium für Ernährung Landwirtschaft und Verbraucherschutz) (2009): Auszahlungspreis für Milch in Deutschland, Daten \& Tabellen. URL: $\quad$ http://www.bmelv-statistik.de/index.php?id=139 \&stw=Milcherzeugerpreise, Abrufdatum: 18.11.2009. Berlin.

BosCHET, M. (2009): Ausrichtung der genossenschaftlichen Milchwirtschaft an eine veränderte gemeinsame Agrarpolitik. Vortrag auf der Tagung für die genossenschaftliche Milchwirtschaft in Kassel.

BundESKARTELLAMT (2009a): Fallbericht: 1. Zusammenschluss Humana Milchunion eG und Milchveredelung Niedergrafschaft, Zusammenschluss Nordmilch und Dargun; 2. Allgemeines Marktumfeld der Milchwirtschaft und aktuelle Fragen zu den rechtlichen Rahmenbedingungen, 27.07.2009. Bonn.

-(2009b): Sektoruntersuchung Milch, Zwischenbericht Dezember 2009. Bonn.

CORDES, H. (2009): Nordmilch gewinnt. In: Netzwerk: Magazin für Kooperation und Management 1, Juli 2009: 50-51.

CWT (Cooperatives Working Together) (2009): News - October 2009. URL: http://www.cwt.coop. Arlington, USA.

DAIRY AUSTRALIA (2009a): Dairy 2009: Situation and Outlook. Victoria, Australia.

- (2009b): Dairy 2009: Situation and Outlook - October 2009 Update. Victoria, Australia.

DBV (Deutscher Bauernverband) (2009): Ergebnisse der Milchquotenbörsen, Termine 2008-2009. URL: http://www.bauernverband.de/index.php?redid=159923, Abrufdatum: 17.11.2009. Berlin.

DEUTSCHE MilchWIRTSCHAFT (2009): Weltmilchgipfel 2009 in Berlin. In: Deutsche Milchwirtschaft 60 (21) 812-819.

DEUTSCHE MOLKEREI ZEITUNG (2009): Viele Industriekunden für die Milchwirtschaft dauerhaft verloren? In: Deutsche Molkerei Zeitung: 8.

EU-AMtSBLATT (1999): Verordnung (EG) Nr. 1255/1999 des Rates vom 17. Mai 1999 über die gemeinsame Marktorganisation für Milch und Milcherzeugnisse. In: Amtsblatt Nr. L 160/48 vom 26.6.1999. Brüssel.

- (2009): Verordnung (EG) Nr. 1038/2009 des Rates vom 19. Oktober 2009 zur Abweichung von der Verordnung (EG) Nr. 1234/2007 (über die einheitliche GMO) hinsichtlich der Interventionszeiträume 2009 und 2010 für Butter und Magermilchpulver. In: Amtsblatt Nr. 288/1 vom 4.11.2009. Brüssel.

EU-KoMMISSION (2009a): Mitteilung der Kommission an den Rat: Die Lage auf dem Milchmarkt im Jahr 2009. KOM(2009) 385. Brüssel.

- (2009b): Pressemitteilung: Kommission verabschiedet Nahrungsmittelhilfeprogramm 2010. IP/09/1779. Brüssel.
- (2009c): Communication \& Information Resource Centre Administrator (CIRCA): Milk Management Committee Statistics. URL: http://circa.europa.eu/, updated: 11.12.2009. Brüssel.

-(2009d): Analysis of price transmission along the food supply chain in the EU. Com (2009) 591. Brüssel.

EUROSTAT (2008): From grass to glass; a look at the dairy chain. Statistics in focus 76/2008. Luxembourg.

- (2009): EUROSTAT Database. URL: http://epp.eurostat. ec. europa.eu/, Abrufdatum: 10.12.2009. Luxemburg.

FAHLbusch, M., A. BAHr, B. BRÜMMER und A. SPILler (2009): Der Markt für Milch und Milcherzeugnisse. In: Agrarwirtschaft 58 (1): 36-52.

FAO (Food and Agricultural Organization of the United Nations) (2009): FAOSTAT Database. URL: http://faostat.fao.org/, Abrufdatum: 8.12.2009. Rom.

GoulD, B.W. (2009): Dairy Data, Homepage of the Dairy Marketing and Risk Management Program. URL: http://future.aae.wisc.edu/. Department of Agricultural and Applied Economics, University of Wisconsin, Madison, USA.

GTIS (Global Trade Information Services) (2009): Global Trade Atlas. URL: http://www.gtis.com. Columbia, USA.

Hanau, A. (1927): Die Prognose der Schweinepreise. In: Vierteljahrshefte zur Konjunkturforschung, Sonderheft 7. Institut für Konjunkturforschung, Berlin.

IDM (International Dairy Magazine) (2009a): Global Dairy Companies Top-20. In: International Dairy Magazine 5: 50 .

- (2009b): Dairy-free analogue Cheese. In: International Dairy Magazine 7: 25.

IFH (Institut für Handelsforschung) und BBE (BBE Retail Experts) (2009): Angebots- und Nachfragemacht: Zum Verhältnis von Industrie und Handel, Studie im Auftrag des Hauptverbands des Deutschen Einzelhandels. Köln.

INDERHEES, P. (2009): Zukünftige Perspektiven für Molkereien und Milcherzeuger in Norddeutschland. In: Deutsche Milchwirtschaft 60 (15): 548-549.

JANSHEN, R. (2009): Joint Venture Nord-Contor. Vortrag im Rahmen der Agrarmarketingtage, 07.-08.12.2009, Berlin.

KECKL, G. (2009): 140 Jahre Marktpartnerschaft Butter und Margarine. In: Deutsche Milchwirtschaft 60 (19). Gelsenkirchen.

LANDESÄMTER FÜR LEBENSMITTELÜBERWACHUNG:

BAYERN (2008): LGL Jahresbericht 2008. URL: http://www.lgl.bayern.de/publikationen/doc/jahresberichte /2008/kapitel_3.pdf, Seite: 52, Abrufdatum: 17.12.2009.

NIEDERSACHSEN (2008): Untersuchungsergebnisse 2008 für Käse. URL: http://www.laves.niedersachsen.de/ master/C55297714_L20_D0_I826_h1.html, Abrufdatum: 17.12.2009.

NORDRHEIN-WESTFALEN (2008): Jahresbericht 2008. URL: http://www.cvua-mel.de/images/stories/cvuameldocs/ Jahresbericht_2008.pdf, Abrufdatum: 17.12.2009.

LASSEN, B., F. ISERMEYER und C. FRIEDRICH (2009): Regionaler Strukturwandel in der deutschen Milchwirtschaft. In: Agrarwirtschaft 58 (5): 238-247.

LeBensmitTelzeitung (2009a): EU soll Problem mit Imitaten lösen. In: Lebensmittelzeitung 61 (35), August 2009.

- (2009b): Marketer entdecken den POS neu. In: Lebensmittelzeitung 61 (35): 40, August 2009. 
- (2009c): Milchpreise ziehen an. In: Lebensmittelzeitung 61 (44): 21, Oktober 2009.

- (2009d): Markt für H-Milch stark konzentriert. In: Lebensmittelzeitung 61 (39), September 2009.

- (2009e): Nordmilch hat das Nachsehen. In: Lebensmittelzeitung 61 (45): 27, November 2009.

MilchindUSTRIEVERBAND (2009): Einblick, Analysen und Perspektiven, Geschäftsbericht 2008/2009. MilchIndustrie Verband e.V., Berlin.

MEIER, W. (2009): Erfolgreiche Exportstrategien am Beispiel der Milch-Union Hocheifel eG. Vortrag im Rahmen der Agrarmarketingtage 07.-08.12.2009, Berlin.

MeINE MiLCH (2009): Milchprodukte im Jahr 2008: Private Nachfrage und Preisschwankungen. URL: http://www.meine-milch.de/artikel/milchprodukte-imjahr-2008-private-nachfrage-und-preisschwankungen, Abrufdatum: 10.12.2009.

MEYER, U. und H. WeINDLMAIER (1988): Die Einstellung der Verbraucher in der Bundesrepublik Deutschland zu Milchimitaten. Milchmarketing Ratgeber Nr. 4, RemagenRolandseck, Bonn.

MOLKEREIINDUSTRIE (2009): Top 10 Molkereien in Deutschland. In: Molkereiindustrie Oktober 2009: 32.

NMPF (National Milk Producers Federation) (2009): News Release: CWT accepts 154 bids, 26.000 cows in third round of 2009. URL: http://www.nmpf.org. Arlington, USA.

RENTENBANK (2009a): Rentenbank startbereit für Liquiditätshilfe der Bundesländer. URL: http://www.renten bank.de/cms/beitrag/10013726/273572/Rentenbank_start bereit_fuer_Liquiditaetshilfe_der_Bundeslaender.html, Abrufdatum: 17.11.2009. Frankfurt am Main.

- (2009b): Rentenbank hat bereits ein Drittel der Liquiditätshilfedarlehen zugesagt. URL: http://www.renten bank.de/cms/beitrag/10013898/273572/Rentenbank_hat _bereits_ein_Drittel_der.html, Abrufdatum: 17.11.2009. $\overline{\text { Frankfurt am Main. }}$

Rothfuß, K., A. ZondLer, B. ENGLer und R. DOLUSChitz (2009): Entwicklung der Milcherzeugung in ausgewählten Regionen Baden-Württembergs - Prognose und kritische Entscheidungsfaktoren. In: Agrarwirtschaft 58 (5): 248-258.

Steffen, N., S. Schlecht und A. SpILler (2009): Ausgestaltung von Milchlieferverträgen nach der Quote. Diskussionspapier der Universität Göttingen, Department für Agrarökonomie und Rurale Entwicklung, Nummer: 0909.
USDA-AMS (United States Department of Agriculture Agricultural Marketing Service) (2010): International Dairy Market News Reports. Washington.

USDA-FAS (United States Department of Agriculture Foreign Agricultural Service) (2009a): Dairy: World Markets and Trade. Circular Series, FD 1-09, August 2009. Washington.

- (2009b): Dairy: World Markets and Trade. Circular Series, FD 2-09, December 2009. Washington.

- (2009c): Russian Federation, Dairy and Products: Import Duties Raised on Milk and Butter 2009. Global Agriculture Information Network (GAIN) Report Number RS9006, 07.02.2009. Washington.

- (2009d): Russian Federation, Dairy and Products: Import Duties Raised on Infant Formula 2009. Global Agriculture Information Network (GAIN) Report Number RS9019, 18.03.2009. Washington.

- (2009e): Mexico, Dairy and Products Semi-Annual 2009. Global Agriculture Information Network (GAIN) Report Number MX9031, 15.05.2009. Washington.

- (2009f): News Releases: USDA announces initial 20092010 Allocations for Dairy Export Incentive Program. Release No. 0289.09. Washington.

USDA-FSA (United States Department of Agriculture Farm Service Agency) (2008): PS-18R Weekly Purchase Report December 1, 2008 - Dairy Products. Washington.

WEINDLMAIER, H. und T. OBERSOJER (2009): Optionen für die einzelbetriebliche Mengensteuerung am Milchmarkt nach dem Ende der Quotenregelung 2015 - Ansatzpunkte und Problembereiche. In: AGRA-EUROPE 46/09 vom 09. November 2009, Dokumentation: 1-11.

ZMP (Zentrale Markt- und Preisberichtstelle) (verschiedene Jahrgänge): ZMP Marktbilanz Milch. Bonn.

- (1995): ZMP Marktbilanz Milch. Bonn.

- (2000): ZMP Marktbilanz Milch. Bonn.

- (2007): ZMP Marktbilanz Milch. Bonn.

- (2008): ZMP Marktbilanz Milch. Bonn.

\section{Kontaktautor:}

\section{Prof. Dr. ACHIM SPILler}

Georg-August-Universität Göttingen

Department für Agrarökonomie und Rurale Entwicklung Platz der Göttinger Siegen 5, 37073 Göttingen

E-Mail: a.spiller@agr.uni-goettingen.de 